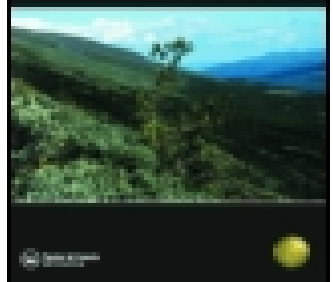

\title{
XV. Enumeration of Polyporus
}

\author{
M. C. Cooke M.A. LL.D.
}

To cite this article: M. C. Cooke M.A. LL.D. (1879) XV. Enumeration of Polyporus, Transactions of the Botanical Society of Edinburgh, 13:1-4, 131-159, DOI: $10.1080 / 03746607909468768$

To link to this article: http://dx.doi.org/10.1080/03746607909468768

册 Published online: 01 Dec 2010.

Submit your article to this journal $\widetilde{ }$

LII Article views: 4

Q View related articles ๘

4 Citing articles: 1 View citing articles ๘ 
cxamined the flowers minutely, but I rather think they were abnormal, as the stamens were very irregular in length." I am not quite sure whether Mr M'Nab's allusion to "thirty years ago" implies his belief that the climate of Scotland has in the interval altered for the worse, or that the imported New Zealand Kowhai has become deteriorated in strength.

\section{Enumeration of Polyporus. By M. C. Cookr, M.A., LL.D. \\ (Read 2d July 1878.)}

The number of species in the genus Polyporus having become so large, I found it necessary to construct for $\mathrm{my}$ own use an alphabetical catalogue, but upon the suggestion that it would also be useful to others, I made the addition of geographical distribution, and references to technical descriptions. With these accessions I submit the list to the Society, for the use of its members, reserving any observations which I may deem it desirable to make, on comparative distribution, for a future occasion, when the list may be consulted in verification. It is only necessary to observe that such synonyms as have been included are indicated by the use of a different type. No critical opinion has been ventured as to the value of the species included.

\section{POLYPORUS, Fr.}

abietinus, DC., Fl., Fr.=amorphus, $F r$. anictisus, Fr., Hym. Eur., 569 (Inodermei), Arctic America, U. States, Europe.

Amxoryis, Lev., Ann. Sci. Nat., 1844, 186 (Ino.), Java.

ABRU PTUs, Berk., Linn. Journ. xvi. 42 (Anodermei), Aru.

ACAxthoides (Bull), Fr., Hym. Eur., 540 (Merisma), Europe.

accrinus, Opiz seznam, 137, sp. dubia.

ACICULA, B. \& C., Linn. Journ. x. 304 (Mesopus), Cuba.

Actrioboles, Mont., Syll., 166 (Ino.), Guiana.

Accleaxs, Berk., Hook. Journ., 1856, 199 (Ino.), Brazil.

accleatos, Lev., Ann. Sci. Nat., 1846, 137 (Ino.), Java.

aculeatus, Mont., Ann. Sci. Nat., 1840, $205=$ Hexagona aculeata.

accpunctates, $B$. \& Br., Linn. Journ. xiv. 52 (Resup.), Ceylon.

a Damaxtixes, Berk., Hook. Journ., 1852, 141 (Placo.), India.

An.sir, B. =dilatatus, B. (Pleur.), Australia, Ceylon.

Anroses, B. \& Br., Fr., Hym. Eur., 550 (Ano.), Europe.

ADesres, Fr., Hym. "Eur., 549 (Ano.), Cuba, India, N. Zealand, U. States, Tahiti, Japan, Brit. N. America, Mexico, Sandwich Isles, Europe.

TRAXS. BOT. SOC. VOL. XIII. 
Eaerita, Fr., Nova Symb., 55 (Placo.), Mexico.

muduaxs, B. \& C., Linn. Jour. x. 304 (Mesopus), Cuba.

escoli (Schw.), Fr., Epicr., 454 (Ano.), U. States.

affinis (Nees), Fr., Epicr., 445 (Pleur.), Australia, Brazil, Aru, Philippines, Java, Admiralty Isles, Sumatra, Nicobar, Pegu.

AfZeLII, Fr, Epicr., 461 (Placo.), Guinea. agariceus, Berk., Ann. Nat. Hist. $=$ arcularius, Fr. agilis, Vivian, t. $57=$ virellus, $\boldsymbol{F r}$.

Alabame, B. \& Cke., Grev. vi. 130 (Resup.), U. States. albido-fuscus, Secr. Myc. = petaloides, Fr.

$\triangle$ LBIDUs, Fr., Hym. Eur., 567 (Ino.), Europe. albidus, Sow., Fung., t. $226=$ cæsius, $F r$. albidus, Wahllog., Lapp. = pallescens, $F r$.

albocervinus, Berk., Hook. Journ., 1856, 234 (Ino.), Brazil, Ceylon.

albogilvus, B. \& C., Linn. Journ., x. 308 (Ano.), Cuba.

albomarginatus, Zipp., Ann. Sci. Nat., 1844, 191 (Placo.), Java.

albostrgids, B. \& C., Linn. Journ. x. 309 (Ano.), Cuba. albus, Bolt., Fung., t. $58=$ salignus, $F r$.

albus (Huds.), Fr., Hym. Eur., 549 (Ano.), Europe. albus, Schœff, t. $314=$ borealis, $F r$.

alligatus, Fr., Hym. Eur., 543 (Merisma), Europe.

ALPINCs, Sauter, Hedwigia, 1876, 33 (Pleur.), Europe.

alutaceus, Fr., Hym. Eur., 545 (Ano.), Europe, U. States. alutaceus, Rostk., t. 27 =destructor, $F r$.

alveolarids, Fr., Epic., 431 (Mes.), U. States, Costa Rica. alveolarius, Rostk., 28, t. $15=$ arcularius, Fr.? amaricans, Pers., Syn., 531 = imbricatus, $F r$.

Ambornevsis, Fr., Epic., 442 (Pleur.), Amboyna, Mauritius, Ceylon, Tongatabu, Philippines, Singapore, Java, Pegu.

AMORPHUS, Fr., Hym. Eur., 550 (Ano.), U. States, Europe.

amygdalinus, B. \& Rav., Grev. i. 49 (Pleur,), U. States.

ANax, Berk., Grevillea, i. 49 (Merisma), U. States.

ANEBUS, Berk., Hook. Journ., 1847, 504 (Ino.), Ceylon, Pegu. aneirinus, (Somm.), Fr., Hym. Eur., $575=$ Trametes aneirina, C. \& Q.

anectoporus, $B . \&$. C., Limn. Journ. x. 318 (Res.), Cuba. angulatus, Pers., Myc. Eur. ii. $72=$ zonatus, $\boldsymbol{F r}$.

Axgestus, Berk., Fl. Tasm., 253 (Ano.), Tasmania. anisopilus, Lev., Ann. Sci. Nat, 1844, 191=pubescens, Fr.

ANisopords, Mont., Syll., 156 (Pleur.).

annosus, Fr., Hym. Eur., 564 (Placo.), Cuba, U. States, Europe. annosus, Fr., El., $106=$ roburneus, $F r$.

annulatus, Jungl., Fl. Jav., $53=$ annularis, Fr.

annolaris, Fr., Nova Symb., 36 (Ino.), Java.

anthelminticds, Berk., Gard. Chron., 1866 (Pleur.), Pegu.

apalUs, Berk., Hook. Journ., 1843, 635 (Mes.), Brazil.

apalus (Lev.), Fr., Hym. Eur., 566 (1848) (Ino.), Europe.

A POPHYSATUS, Rostk., 27, t. 4 (Mes.), Europe.

a PPendiculatus, $B$. \& Br., Linn. Journ. xiv. 48 (Ano.), Ceylon.

applanatos (P.), Fr., Hym. Eur., 557 (Placo.), Brazil, U. States, Pegu, Europe.

appositus, Lev., Ann. Sci. Nat., 1846; 141 (Resup.), Java.

APricus, Berk., Fl. Tasm. (Resup.), Tasmania.

aratus, Berk., Lim. Journ. xvi. $\mathbf{5 3}$ (Ino.), Lord Howe's Island.

Archeri, Berk., Fl., Tasm., 255 (Resup.), Tasmania.

ARCTICUs, Fr., Epicr., 479, (Ino.), Kantschatka.

arcolarios (B.), Fr., Hym. Eur., 526 (Mes.), India, Ceylon, Cnba, Bermuda, U. States, Australia, N. Zealand, Surinam, Java, Cape, Europe. 
ARExaries, Klotsch., Fr., Epicr., 487 (Resup.), India.

ARricolon, B. \& C., Linn. Journ. x. 315 (Ino.), Cuba. argcuteus, Ehr., Bor., p. $27=$ adustus, Fr.

arcillaceus, Coolie, Grov. vii. I (Resup.) U. States. argyraceus, Pers., Myc. Eur. ii. $73=$ versicolor, Fr.

armeniacus, Berk., Eng. Fl., v. $147=$ amorphus, Fr.

amMexiacus, Berk., Hook. Journ., 1856, 197 (Ano.) Brazil, Aru.

AISIExICOLOR, B. \& C., Linn. Journ. x. 315 (Ino.) Cuba.

artcmidorus, Lenz., f. $\mathbf{4 3}=$ confluens, $F r$.

antexsis, Berk., Linn. Journ. xvi. 43 (Placo.), Aru.

AscoBoloIDEs, Berk., Linn. Journ. xiii. 162 (Áno.), Australia.

AsPER, Jungh., Fl. Jav., 60 (Pleur.), Java.

A $\div$ Prellus (Lev.), Fr., Hym. Eur., 524 (Mes.), Europe.

atrates, Fr., Nova Symb., 124 (Pleur.), Mexico.

atropdrpureus, Berk., Hook. Journ., 1856, 194 (Pleur.) Brazil.

Atroumbrinus, Berk., Hook. Journ., 1856, 199 (Placo.), Brazil.

atTexuatus, Peck., 26th Report, N.Y., 70 (Resup.), U. States.

atrpes, Lev., Ann. Sci. Nat., 1844, 184 (Pleur), Java, Guadeloupe.

A crerianus, Mont., Cuba, t. xvi. f. 1. (Placo.), Cuba, Brazil, Guiana, Mexico, Bermuda.

acgustus, Berk., Hook. Journ., 1856, 143 (Mes.), Brazil.

acraxifacus, Peck., 26th Report, N.Y., 69 (Ino.), U. States.

aurantiacus, Rostk., 4 , t. $58=$ spongiosus, Fr.?

alrastiopillens, B. \& C., Grev. i. 53 (Res.), U. States.

aurantius, Schœeff., t. 108, $110=$ confluens, Fr.

aurantizs, Trog., Flora, 1852, $354=$ confluens, Fr.

aureolus, Pers., Myc. Eur. ii. $60=$ amorphus, Fr.

arriculeforMis, Jungh., Ann. Sci. Nat., 1844, 194 (Res.), Java.

Almirorsis, Mont., Syll., 160 (Placo.), Guiana.

acriscalpidm (Pers.), Fr., Epicr., 443 (Pleur.), Brazil.

Acstralis, Fr., Hym. Eur., 556 (Placo.), Cuba, Borneo, Tahiti, Fiji,

Guiana, Brazil, Moluccas, Java, Bermuda, India, Tasmania, N. Zealand,

Yenezuela, Australia, Ceylon, Mauritius, New Hebrides, Aru,

Admiralty Isles, Guadeloupe, Nicobar Islands, Cochin' China, Europe.

azcreus, Fr., Nova Symb., 77 (Ino.), Mexico.

B.sprcs, Berk., Ann. Sci. Nat., 1846, 138 (Ino. ?), Guadeloupe.

lutlius, Weinm., Ross, 311 = varius, Fr.

B.AL.SIIIFERE, Klotsch., Linn., 1833, 486, U. States.

immiatulus, Fr., Nova Symb., 71 (Ino.), U. States.

Mnnb.eformis, $B$. \& C., Grev. i. 53 (Resup.), U. States.

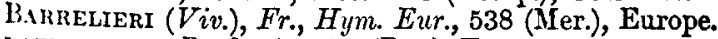

n.t thyponus, Rostk., 4, t. 59 (Res.), Europe.

Peckleri, Berk., Linn. Journ. x. 162 (Ano.), Australia.

Brinarexsis, Berk. Hook. Journ., 1852, 163 (Res.), India.

Bexetostos, Berk., Linn. Joum. xvi. 52 (Res.), Tahiti.

Mrizonos (Whlog.), Fr., Hym. Eur., 554 (Placo.), U. States, Europe.

Brakeley, Fr., Nova Symb., 40 (Mer.), U. States.

Briclisus, Fr., Hym. Eur., 555 (Placo.), U. States, Arctic America,

Europe.

Beyrichii, Fr., Linn. v. $578=$ Trametes.

bilhulus, Pers., Myc. Eur., $135=$ medulla-panis, Fr.

BIColor, Jungh., Fl. Java, 54 (Ino.), Java.

nrixis, Fr., Hym. Eur., 529 (Mes.), Europe.

Bifonmis (Klot.), Fr., Epic., 475 (Ino.), U. States, Arctic America, Mexico.

ninRetus, Kalch., Hedwigia, xv. 114 (Ano.), Australia. 
Bistratosus, B. \& Cke., Limn Journ. xv. 384 (Res.), Brazil.

BIValvis (Pers.), Fr., Epic., 480 (Ino.), Borneo.

Blanchetianus, Mont., Syll., 155 (Pleur.), Brazil.

Blepharistoma, $B . \& B$., Ann. N. Hist. No. 1434 (Res.), Europe.

Blomer, Lev., Ann. Sci. Nat., 1844, 185 (Pleur.), Java.

Blytti, Fr., Hym. Eur., 571 (Res.), Europe.

Boltoni, Rostk. 28, t. $24=$ varius, $F r$.

bombycinus, Wirtg., Flora, $1835=$ Wirtgeni, Fr.

bombycinus, Fr., Hym. Eur., $575=$ Trametes.

Bonplandiands, Lev., Ann. Sci. Nat., 1846, 301 Paraguay.

Borealis, Fr., Hym. Eur., 552 (Ano.), U. States, N. Zealand, Siberia, Europe.

Botryoldes, Lev., Ann. Sci. Nat., 1846, 128 (Mer.)

botulatus, Secr. Myc. = fulvus, Fr.

Boccheands, Fr., Hym. Eur., 533 (Pleur.), U. States, Europe.

BRAChYPoRUs, Mont., Syll., 162 (Pleur.), Cayenne.

Brachypus, Lev., Ann. Sci. Nat., 1846, 127 (Pleur.), Guadeloupe.

Braunir, Rabh., Fungi Eur., 2005 (Ino.), Europe.

Broomer, Rabh., Fungi Eur., 2004 (Res.), Europe.

Bromalis, Fr., Hym. Eur., 526 (Mes.), U. States, Cape, Europe.

brumalis, Rosk. iv. t. $5=$ ciliatus, Fr.

BRoxineoleucus, $B$., Hook. Journ. v. 4 (Ino.), Tasmania.

Bruxneouds, B., Sill. Journ., 1851, 94 (Pleur.), Philippines, Samoan group, Java.

BRONNEOPICTUS, B., Hook. Journ., 1856, 176 (Pleur.), Brazil, Arracan.

brunneus, Pers., Myc. Eur. ii. $95=$ violaceus, Fr.?

Bullosus, Fr., Hym. Eur., 579 (Res.), Europe.

BUlbipes, Fr., Plant. Preiss., p. 135 (Mes.), Australia, Mexico.

byrsinus, Mont., Cuba, t. xv. f. $3=$ Trametes occidentalis, Fr.

byssinus, Pers. Myc. Eur. ii. $122=$ dubia.

byssina, Secr. (sub Poria) = reticulatus, Fr.

Byssogenos, Jungh., Fl. Java, 43 (Res.), Java.

Cadaverirvos (Schulz.), Fr., Hym. Eur., 544 (Mer.), Europe.

CessiUs, Fr. IIym. Eur., 547 (Ano.), U. States, Europe. calceolus, Bull, Champ. = elegans, Fr.

calceus, B. \& Br., Linn. Journ. xiv. 55 (Res.), Ceylon.

Calcigenus, B., Hook. Joum., 1843, 636 (Mfes.), Brazil.

calcitratus, $B$. \& $C$., Linn. Journ. x. 314 (Ino.), Cuba.

caliginosus, B., Linn. Journ. xvi. 46 (Placo.), Philippines, Admiralty Islands.

CatimMorphto, Lev., Ann. Sci. Nat., 1846, 132 (Ino.), Madagascar.

Callochrous, Lev., Ann. Sci. Nat., 1844, 181 (Mes.)

Callosus, Fr., Hym. Eur., 577 (Res.), U. States, Ceylon, Europe.

calvescens, B., Ann. Nat. Hist., 1839, 390 (Ano.), U. States.

caserarius, B., Hook. Journ., 1856, 175 (Mes.), Brazil.

CaMpbelli, B., Hook. Journ., 1854, 228 (Mes), India.

CAMPYLOPORUs, Mont., Syll., 163 (Ino.), Guiana.

CAMPYLCS, Berk., Fl. Tasm., 252 (Ano.), Tasmania.

canalium (Lour.), Fr., Sys. Mfyc. i. 352 (Mes.), Cochin China.

Candidissrimos, Schw., Amer. Bor., 468 (Res.), U. States.

CaNdidolus, Lev., Ann. Sci. Nat., 1846, 301 (Ano.)

candidus, Pers., Myc. Eur., t. 15, f. $2=$ chioneus, Fr.

caNdidUs, Fr., Hym. Eur., 541 (Mer.), Europe.

caperatus, B., Ann. N. Hist., 1839, 391 (Ino.), Cuba, Philippines,

Guiana, Brazil, India, Ceylon, Martinique, Centr. Amer., Mauritius.

captiosus, Mont., Ann. Sci. Nat, 1847, 170=Trametes. 
cupcuxcs, Mont., Ann. Sci. Nat. v. 369 (Placo.), Brazil. Cikboxaceus, B. \& C., Linn. Joum. x. 317 (Res.), Cuba. cinboxarius, Fr., Hym. Eur., 532 (Mes.), Europe.

Carmichaelianus, Grev., t. $224=$ Merulius. curnenfulves, Berk. =omalophilus, Mont.

cinseopay.lexs, B., Hook. Joum., 1856, 237 (Res.), Brazil, Cuba.

c.rnxeus (Nees), Fr., Hym. Eur., 563 (Placo.), Cuba, Brazil, U. States, Java, Australia, West Africa; Europe.

Carolinensis, Berk., Hook. Journ., $1849=$ biformis, Fr.

carpineus, Sow., t. $231=$ adustus, Fr. var.

cartilaginets, $B$. \& Br., Linn. Journ. xiv. 49 (Placo.), Ceylon.

CARTe, Schw., Amer. Bor., 441 (Res.), U. States.

casces, Fr., Nova Symb., 72 (Ino.), Costa Rica.

casearios, Fr., Hym. Eur., 541 (Mer.), Europe.

c.sssiecolor, B., Hook. Journ., 1856, 171 (Mes.), Brazil.

castaneus, Rostk. 4, t. $47=$ fuscatus, Fr.

castaxeus, Fr., Hym. Eur., 564 (Placo.), Europe.

catervatus, B., Fl. New Zeal. (Mes.), New Zealand.

caudicinus, Scop. $=$ sulphureus, $F r$.

caversolosus, B., Hook. Journ., 1856, 236 (Res.), Brazil, Cuba. cellaris, Desm., Crypt., No. $72=$ igniarius, Fr.

cellulosus, Whlbg., Suec. $=$ vulgaris, Fr.

cephalotes, Pers., Myc. Eur. ii. $118=$ dubia.

cerasi, Rostk., t. 61, uncertain.

(eratoxia, Fr., Hym. Eur., 552 (Ano.), Europe.

crenes, B., Hook. Journ., 1852, 163 (Res.), India.

cenifluus, B. \& C., Grev. i. 50 (Ano.), U. States.

CERvinogilvus, Jungh., Fl. Jav., 45 (Ino.), Java, Nicobar.

(ERvixonitens, S. Ann. N. Hist. xvi. 433 (Pleur.), U. States. cervinoplumbeus, Jungh. (fide Lev.)= Hexagona.

ccrinus, Pers., Myc. Eur. ii. $87=$ Trametes mollis, Fr.

CERvives (Schw.), Fr., Epic., 474 (Ino.), U. States.

chalceus, Cke. (cupreus, Fr., non B.), (Placo.), Guinea.

cunataceus, B. \& C., Grev. i. 53 (Ino.), U. Stater.

cillessis, Fr., Nova Symb., 47 (Placo.), Chile, Australia, Cape.

chroxeus, Fr., Hym. Eur., 546 (Ano.), U. States, Europe.

chinomaticus, B. \& Cke., Linn. Journ. xv. 384 (Res.), Brazil.

chrrseds, Lev., Ann. Sci. Nat., 1846, 301 (Res.), New Granada.

Cinrsites, B., Hook. Journ., 1856, 233 (Ino.), Brazil, Cuba.

Chrisoloma, Fr., Hym. Eur., 574 (Res.), Europe.

Currsobaphus, $B$. \& $C$., Grev. i. 53 (Res.), U. States.

Chrssoleucos, Kalch., Grev. iv. 74 (Ino.), Australia.

cichoraceus, Fr., Nova Sym., 76 (Ino.), Philippines, Australia, Ceylon.

ciliatus, Fr., Hym. Eur., 527 (Mes.), U. States, Europe.

cilicioldes, Fr., Nova Symb., 71 (Ino.), Indian Archipelago, Philippines

crictus, Berk., Outl., 250 (Res.), Europe.

cixeraceus, Lev., Ann. Sci. Nat., 1846, 139 (Ino.), India.

cinerascens, Lev., Ann. Sci. Nat., 1844, $184=$ Leveillei, Cke.

Cinerascens, Fr., Epic., 481 (Ino.), U. States, Pegu.

cisereofuscts, Curr., Linn. Trans., 1876, 124 (Placo), Pegu.

cinereolutescens, Pers., Myc. Eur. ï. 205=ravidus, Fr.

Cixerelids, Lev., Ann. Sci. Nat., 1246, 140 sub cinereus (Ino.), Brazil, U. States.

cixereus, Schwz, Amer. Bor., 440 (Res.), U. States.

civgulatcs, Fr., Epior., 476 (Ino.), Brazil, India, West Indies.

cinnabarinus, Fr., Sys. Myc. i. $371=$ Trametes.

(IxxaMomets (Trog.), Fr., Hym. Eur., 561 (Placo.), Europe. 
CIRcinatus, Fr., Hym. Eur., 530 (Ifes.), Europe. cirritrerus, B. \& C., Linn. Journ. x. 314 (Ino.), Cuba. citreus, B., Linn. Journ., xiii. 162 (Ano.), Australia. CItrinellus, B. \& C., in Curtis Cat. (Placo.), U. States. citrinus, Planer = sulfureus, $F r$.

cladonia, B., Hook. Journ., 1845, 61 = bulbipes, Fr. cladotrichus, B. \& C., Linn. Jour. x. 309 (Ano.), Cuba. clathratus, B. \& C., Grev. i. 54 (Res.), U. States. coccineus, Fr., Nova Symb., 51 (Placo.), Coral Islands. cochlear, Nees, Act. Nat. Cur. xiii. 20) (Pleur.), Java, Aru. condleororus, Peck., 26th Report, N. Y., 68 (Mes.), U. States. cordleds (Schum.), Fr., Hym. Eur., 549 (Ano.) Europe. Coffeatus, B., Ann. Nat. Hist., 1839, 385 (Pleur.), St Vincent. comorens, Lev., Ann. Sci. Nat., 1846, 131 (Ino.), Java. cullabefactus, B. \& Br., Ann. Nat. Hist., 1432 (Res.), Europe. Collabens, Fr., Hym. Eur., 572 (Res.), Europe.

Colensoi, Berk., Fl. New Zeal. (Mer.), New Zealand. colliculosus, Pers., Myc. Eur. ii. 163=tuberculosus, Fr. cocossus, Fr., Nova Symb., 40 (Placo.), Costa Rica. colombiensis, Berk., Hook. Journ., 1842, 454 (Mes.), Columbia River. comatus, Fr., Nova Symb., 75 (Ino.), Costa Rica.

cosipressus, B., Hook. Journ., 1845, 53 (Ano.), Australia. concentricus, Schum. = adustus, Fr.

conchatus, Fr., Hym. Eur., 560 (Placo.), Australia, U. States, Europe. conchatus, Quel. Jur. t. 17, f. $5=$ pectinatus, $K l$.

Conchifer (Schw.), Fr., Epicr., 463 (Placo.), U. States. concinnus, Fr., Epic., 436 (Mes.), Tropical Africa, Guinea. Concresceis, Mont. Syll., 166 (Ino.), Juan Fernandez. CONFERTds, Lev., Ann. Sci. Nat., 1844, 187 (Ino.), Java. confluens, Fr., Hym. Eur., 539 (Mes.), U. States, Europe. confiuens, Rostk., 4, t. 34=resinosus, Fr. confluens, Schum., p. $378=$ perennis, $F r$.

conglobatus, $B$., Hook. Journ., 1845, 303, U. States. connatus, Weinm. Ross., $332=$ connatus, Fr.?

connatus, Fr., Hym. Eur., 563 (Placo.), Tahiti, Europe. connatus, Schwz., Ann. Bor. $=$ focicola, $B . \& C$.

connexus, Lev., Ann. Sci. Nat., 1846, 135 (Ino.), Brazil. contiauus, Fr., Bym. Eur., 571 (Res.), Ceylon, U. States, Martinique, Europe.

contractos, B., Hook. Journ., 1847, 503 (Placo.), Ceylon.

Convolutes, Lev., Ann. Sci. Nat., 1844, 186 (Ino.), Java. coriaceus, Lev., Ann. Sci. Nat., 1846, 137 (Ino.), India. coriaceus, Bull, t. $28=$ perennis, $F r$.

CORIUM, B., Hook. Journ., 1852, 163 (Ino.), India. coronatus, Rostk., 28, t. $27=$ Boucheanus, Fr.

conrivalis, Berk., Linn. Journ. xiii. 162 (Ano.), Australia corrugatus, Pers., in Freyc. Voy. = scabrosus, Fr.

corrogatus, Lev., Ann. Sci. Nat., 1846, 136 (Ino.), Java. corrogis, Fr., Hym. Eur., 536 (Pleur.), Europe. corrdars, Fr., Nova Symb., 66 (Ino.), West Indies. Corrobcans, Fr., Hym. Eur., 551 (Ano.), Europe. CORTICoLA, Fr., Hym. Eur., 580 (Res.), Australia, Brazil, U. States, Europe.

CORYlinus (Viv.), Fr., Hym. Eur., 528 (Mes.), Europe. Chassipes, Curr., Linn. Trans., 1876, 122 (Mes.), Pegu. CRAssus, Fr., Hym. Eur., 543 (Mer.), Europe.

Craterellos, B. \& C., Limn. Journ. x. 305 (Mes.), Cuba. 
Cremor, B. \& C., Grev. i. 54 (Res.), U. States.

(REMORICOLOR, B., Hook. Journ., 1851, 79 (Mes.), India. crexatus, B., Ann. Nat. Hist., 1843, 372 (Pleur.), Ceylon, Java, Sumatra. critatus, Berk. (= Ravenalii, B. \& C.), (Pleur.), U. States. cribrosus, Pers., Myc. Eur., ii. $96=$ dubia.

crispus, Fr., Hym. Eur., 550 (Ano.), India, U. States, Europe.

Cristatus, Fr., Hym. Eur., 539 (Mer.), U. States, Europe. cristatus, Schœff., t. $316,317=$ lobatus, $F r$.

cristula, (K1.), Berk., Ann. Nat. Hist., 1839, 387 = cinnabarinus, Fr. ?

crocatus, Fr., Epic., 477 (Ino.), U. States, Mexico. croceus, Karst., Fin. Poly., $39=$ contiguus, Fr.

croceus, (P.), Fr., Hym. Eur., 548 (Ano.), Europe.

CROCICOLOR, B. \& Cke., Grev. vi. (Ino.), U. States.

crociponos, $B$. \& $C$., in Curtis Cat. (Res.), U. States.

crocitinctus, B. \& C., Linn. Journ. x. 311 (Placo.), Cuba

croentatus, Mont., Syll., 161 (Res.), Guiana, U. States.

cruentus, Pers., Myc. Eur. ii. t. 16, f. $4=$ incarnatus, Fr.

crustaceUs, Lev., Ann. Sci. Nat., 1844, 193 (Res.), Java.

Criptacanthos, Mont., Ann. Sci. Nat. v. 369 (Res.), Brazil.

cryptardm (Bull), Fr., Hym. Eur., 566 (Ino.), U. States, Europe.

cebessrs, Mont., Syll. 160 (Placo.), Cuba.

cocollatus, B. \& C., Grev. i. 51 (Placo.), U. Stateg.

Cumingir, B., Hook. Journ., 1842, 147 (Mes.), Philippines.

cupreoroseus, B., Hook. Journ., 1856, 233 (Ino.), Brazil, Cubs.

cupreus, Fr. Nova Symb. $48=$ chalceus, Cke.

cupreus, B., Ann. Nat. Hist., 1839, 393 (Ino.), India.

COPULAFORMIS, $B$. \& C., Grev. i. 38 (Mes.), U. States.

curtipes, B. \& C., Hook. Journ., 1849, $235=$ Favolus curtipes, B. \& $C$.

Cortisir, Berk., Grev. i. 38 (Pleur.), U. States, Mexico.

coticolaris, Fr., Hym. Eur., 551 (Ano.), U. States, Europe. cuticularis, Whlgb., Suec., $1998=$ vulpinus, $F r$.

cyathiformis, Lev., Ann. Sci. Nat., 1844, 181 (Mles.), New Spain. cyathoides, Quel., Jur., $253=$ vernalis, Fr.

cyathoides (Sow.), Fr., Hym. Eur., 534 (Pleur.), Europe.

crcliscts, Mont., Syll., 164 (Pleur.), Chili.

crclodes, Fr., Nova Sym., 74 (Ino.), W. Indies.

CrPhelloIdes, Fr., Nova. Sym., 72 (Ino.), Mexico.

crTisinus, Berk., Eng. Fl. v. 142 (Placo.), Eurupe.

DaDaleoIdes, B., Ann. Nat. Hist. iii 325 (Res.), Tasmania.

dealbatos, B. \& C., Grev. i. 39 (Pleur.), U. States.

debilis, Walh., Fl. Germ. ii. $60=$ brumalis, Fr.

DEcIPIENs, Schw., Syn. Car., 386 (Ino.), U. States, Mexico.

DECOLOR, B., Hook. Journ., 1856, 195 (Pleur.), Brazil.

Decolorans, Schw., Amer. Bor., 467 (Res.), U. States.

DeCrescens, Zoll., Arch. Neerl. (Ino.), Java.

DEFoRMIs, Fr., Hym. Eur., 536 (Pleur.), Europe.

DEGLUBENs, Berk., Hook. Journ., 1856, 235 (Res.), Brazil.

DEGLUBens, $B$. \& $C$., in Curtis Cat. (Ino.), U. States (uncertain).

DELICATUS, B. \& C. Grev. i. 37 (Mes.), U. States.

Demidoffi, Lev., Demid. Voy. (Placo.), Europe.

DeMIssus, B., Hook. Journ., 1845, 52 (Ano.), Australia.

Dendriticus, Fr., Nova Symb., 37 (Mer.), IIexico, U. States.

Dentipords, Pers., Myc. Eur. ii. 104 (Res.), U. States, Europe.

DEPENDENs, $B$. \& $C$., Grev. i. 37 (Mes.), U. State8.

dermatodes, Lev., Voy. Bon. t. $138=$ Trametes.

Destructor, Fr., Hym. Eur., 547 (Ano.), U. States, Europe. 
DETOAsus, $F r$., Epic, 478 (Ino.), Brozil, Java. Detritus, B., Hook. Joum., 1816, 197 (Ano.), Brazil. diabolices, B., Hook. Joum., 1856, 174 (Mes.), Brazil. Dialespes, Fr., Epic., 456 (Ano.), Guinea. DIbaphus, B. \& C., Grev. i. 36 (Mes.), U. States. DIChrous, Fr., Hym. Eur., 550 (Ano.), N. America, U. States, N. Zealand, Europe.

DickInsir, B., Linn. Journ. xvi. 50 (Pleur.), Japan.

Dictyopos, Mont. Syll. 155 (Pleur.), Cape York, Juan Fernandez.

Dietrichseni, Fr., Nova Symb., 60 (Pleur.). Brazil, Borabora.

DIfFIssus, Bcrk., Fl. N. Zeal. (Res.), New Zealand.

Diffusus, Fr., Nova Symb., 39 (Ano.), Cuba, Cahu.

Digitalis, B., Hook. Journ., 1852, 139 (Ano.), India.

dilatatus, Lev., Ann. Sci. Nat., 1844, 184 (Pleur.), Java.

dilatatus, B., Hook. Journ., 1846 = Adami, Berk.

DISCIFormis, Lev., Ann. Sci. Nat., 1844, 193 (Res.), Mauritius.

DISCIPES, Berk., Hook. Journ., 1847, 499 (Pleur.), Ceylon.

Discoideus, $B$. \& C., Linn. Journ. X. 305 (Mes.), Cuba.

DISCOLOR (Kl.), Fr., Epicr., 450 (Mer.), Mauritius.

DISPAR, Kalch., Grev. vii. ined. (Ino.), Australia.

Dissectus, Lev., Ann. Sci. Nat., 1846, 139 (Ino.), Chili.

Dissitus, B.\& Br., Linn. Jourm. xiv. 48 (Ano.), Ceylon.

Distortus (Schw.), Fr., Epic., 449 (Mer.), U. States.

DIVERsiporus, $B . \&$ \& $B$., Linn. Journ. xiv. 53 (Res.), Ceylon.

DochMius, B. \& Br., Linn. Journ. xiv. 50 (Placo.), Ceylon.

dolosus, Pers. Myc., Eur. ii. $77=$ abietinus, Fr.

DorCas, B., Ann. Nat. Hist., 1852, 195 (Ano.), st Domingo.

Dozyanus, Lev., Ann. Sci. Nat., 1848, 123 (Ano.), Java.

DrummoNd (Klot.), Fr., Epic., 481 (Ino.), U. States.

DryadeUs, Fr., Hym. Eur., 553 (Placo.), U. States, Europe. dryadeus, Schwz. = scruposus, Fr.

dryadeus, Rostk., 27, t. $9=$ applanatus, $F r$.

DRyinus, B. \& Cke., Grev. vi. 130 (Res.), U. States.

DRyophilus, B., Hook. Journ., 1847, 321 (Ano.), U. States, Mexico.

dubius, Jungh. (fide Leveille) = australis, $F r$.

oubics, B., Ann. Nat. Hist., 1843, 374 (Ino.), Ceylon.

DURUs, Jungh., Fl. Java, 62 (Auo.), Java.

eburneus, Wallr., Fl. Germ. =osseus, Fr.

echinatus, Pers., Myc. Eur. ii. $102=$ dubia.

ECTYPCS, B. \&. C., Grev. i. 52 (Ino.), U. States.

ELACHISTOS, Berk. (=minimus, Rav.) (Res.), U. States.

elatinds, $B$., Hook. Journ., 1852, 140 (Ano.), Indiar

elatus, Lev., Ann. Sci. Nat., 1846, 129 (Placo.), Guadeloupe.

elegans, Bolt., t. $76=$ giganteus, Fr.

elegans, Trog., in Flora = varius, $\mathrm{Fr}$.

eleans, Fr., Hym. Eur., 535 (Pleur.), U. States, Tasmania, Cuba, Admiralty Isles, Europe.

EurisIr, Berk., Grev. vii. 4 (Mer.), U. States.

ELongatus, B., Hook. Journ., 1842, 140 (Ino.), St Domingo, U. States,

Cuba, India, Ceylon, Java, Central America, Australia, Japan,

Philippines, Mexico.

EMolLITUS, Fr., Hym. Eur., 571 (Res.), Europe.

encephalum, Hoffm., Veg. Subt. = dubia.

exdocrocinus, B. Hook. Journ., 1847, 320 (Ano.), U. States.

ENDOPALUS, B., Linn. Journ. xiii. 163 (Placo.), Australia.

ENDOPHCUS, B., Hook. Journ., 1852, 142 (Placo.), India. 
Exdotheius, B., Linn. Journ. xvi. 46 (Placo.), Philippines.

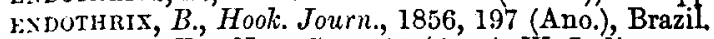
kx Dozoses, Fr., Nova Sym., 38 (Ano.), W. Indies. exteroleucus, Fr., Epic., 468 (Placo.), Chili. cpigca, Lenz., p. $62=$ Schweinitzii, Fr.

elileucUs, Fr., Hym. Eur., 545 (Ano.), U. States, Europe. kPlinteds, B. \& Br., Linn. Journ. xiv. 55 (Res.), Ceylon. erisiltines, $B$. \& Br., Linn. Journ. xiv. 54 (Res.), Ceylon. cpiphyllus, Pers., Obs. ii. $15=$ molluscus, Fr.

epIPTELios, Fr., Mon. ii. 270 (Placo.), Europe. epiranthus, Rostk., t. 30=alutaceus, Fr. ercbescens, Fr., Hym. Eur., 554 (Placo.), Europe. ECCiLYPTI, Kalch., Grev. iv. 73 (Placo.), Australia. edcalyptorum, Fr., Pl. Preiss; 135 (Ano.), Australia. Ecoxysi, Kalch., Enum. ii. 1232 (Placo.), Europe. ecronus (Karst.), Fr., Hym. Eur., 575 (Res.), Europe. evrocephalus, B. \&Br., Linn. Journ. xiv. 48 (Mer.), Ceylon. evolutus, $B$. \& C., Linn. Journ. x. 308 (Ano.), Cuba. Evolvexs, B., Hook. Journ., 1856, 253 (Res.), Brazil exasperatus, Schrad., Spic., 153 = arcularius, Fr.

excurress, B. \& C., Linn. Journ. x. 318 (Res.), Cuba. ExILIs, B., Hook. Journ., 1856, 173 (Mes.), Brazil. Exotepheos, B., Linn. Journ. xvi. 49 (Placo.), Admiralty Islands. Expansus, Fr., Epic., 475 (Ino.), Guinea. extensus, Lev., Ann. Sci. Nat., 1846, 129 (Placo.), Guadeloupe. extendatos, Mont., Syll., 166 (Ino.), Algeria.

fagineus, Schrad., Spic. $161=$ albus, $F r$. FARINeluUs, Fr., Hym. Eur., 579 (Res.), U. States, Europe. fasciatus, Fr., Epic., 470 (Placo.), Brazil, Jamaica. fasciculatus, Schrad., Spic., 154=brumalis, Fr.

rastuosus, Lev., Ann. Sci. Nat., 1846, 134 (Placo.), Java, Brazil, Singapore.

Fatiscens, B. \& Rav., Grev. i. 65 (Res.), U. States.

Favescens, Schwo., Am. Bor., 413 (Res.), U. States.

favillaceds, B. \& O., Grev. i. 53 (Res.), U. States.

favolaris, Fr., Nova Symb., 34 (Mes.), Sambulang. favus, Bull, t. $421=$ Trametes gallica, $F r$.

Feathermanni, Rav., Fungi Amer. = Trametes.

Feer, Fr., Epic., 476 (Ino.), Brazil, Australia, Tasmania, Pegu.

Fendzleri, B. \& C., Linn. Journ. x. 317 (Res.), Venezuela, Cuba.

Fersandesiands, Mont., Syll., 168 (Ino.), Juan Fermandez.

Ferreus, B., Hook. Journ., 1847, 502 (Placo.), Cuba, Ceylon, Brazil. ferreus, Pers., Myc. Eur. ii. $89=$ floccosus, $F r$.

ferrugineus, Jungh. (fide Leveille), = Hasskarlii Lev.

FERruginosds, Fr., Hym. Eur., 571 (Res.), Australia, Cuba, U. States, Europe.

ferruginosus, Rostk., 27, t. $6=$ umbrinus, Fr. fibrillosus, Karst., Fin. Polyp., $30=$ vulpinus, Fr. fibrosoradians, Mont. = nutabilis, $B$.

FIBULA, Fr., Hym. Eur., 567 (Ino.), U. States, Europe.

FISIBRIPORUs, Schw., Am. Bor., 355 (Ano.), U. States.

fimbriatus, Bull, t. 254=pictus, Fr.

Fimbriatus, Fr., Epic., 476 (Ino.), Brazil, Java, Manila.

FIssilis, B. \& C., Grev. i. 50 (Ano.), U. States.

FIssus, B., Hook. Journ., 1847, 318 (Mes.), U. States, Costa Rica.

flabelliformis, Pers, Myc. Eur. ii. 53 = squamosus, F'r. 
flabelliformis, Schff., t. 113=crustatus, $F r$.

Flabelliformis (Klotsch.), Fr., Epic., 444 (Pleur.), India, Ceylon, Mauritius, Borneo, Cula, Peyu, Australia, Lord Howe's Island.

Flabellum, Mont., Cuba, t. xv. f. 2 (Ino.), Cuba.

FlammaNs, $B .$, Hook. Journ., 1852, 139 (Mer.)

flavescens, Rost., 28, t. $23=$ melanopus, $F r$.

Flavescens, Mont., Ann. Sci. Nat. v. 368 (Placo.), Brazil.

FLAVIDUS, B., Hook. Journ., 1852, 161 (Ino.), India.

flavidus, Peck., 29th Report, $68=$ Peckianus, Cke.

Fla vovirens, $B . \&$., Grev. i. 38 (Mes.), U. States.

flavus, Jungh., Fl. Jav., $46=$ Irpex flavus, $K l$.

flavus, Karst., Finl. Polyp. = dubia.

FLEXIPES, Fr., Epicr., 432 (Mes.), Centr. America, Brazil.

FLOCCIPES, Rostk., 28, t. 13 (Mes.), Europe.

Floccosus, Jungh., Fl. Jav., 49 (Ino.), Java.

Floccosus, Fr., Hym. Eur., 572 (Res.), Europe.

Florid a ud, B., Ann. Nat. Hist. x. 376 (Ino.), Brazil, U. States, Aru.

FloRIdeds, B., Hook. Jourm., 1852, 137 (Mes.), India, Pegu.

Focicola, B. \& C. Linn. Jour. x. 305 (Mes.), Cuba, U. States.

FOCKer, Mig., Fungi Rec., 6, Surinam.

FCDAtus, B., Linn. Journ. xvi. 41 (Ano.), Cape York.

foliaceus, Pers., Mzc. Eur. ii. 121 dubia

foliaceus, Jungh., in Herb. = elongatus, B.

fomentarids, Fr., Hym. Eur., 588 (Placo.), India, Australia, Java, U.

States, Europe.

FonniCatus, Fr., Epic., 443 (Pleur.), Brazil.

FRACTIPES, $B$. \& C., Grev. i. 39 (Pleur.), U. States.

Fragilis, Fr., Hym. Eur., 546 (Ano.), U. States, Europe.

FraGilissimus, Mont., Syll., 163 (Pleur.), Guiana.

Fraxineus, Fr., Hym. Eur., 563 (Placo.), Arctic America, U. States, Europe.

Friburgensis, Humb., Frib., $112=$ Trametes odorata, Fr.

Friestr (Klotsch.), Fr., Epicr., 480 (Ino.), N. America, Tasmania, Cape.

Frondosds, Fr., Hym. Eur., 538 (Mer.), U. States, Tasmania, Europe.

frondosus, Schrad., Spic. 21 =intybaceus, $F r$.

frustulatus, Pers., Myc. Eur. ii. $\mathbf{9 1}=$ Trametes serialis, Fr.

FruticuM, B.\& C., Linn. Jour. x. 310 (Ano.), Cuba.

fugax, Pers., Ic. Pict., t. $16=$ reticularis, Fr.

rulaexs, Rostk., 4, t. 63 (Res.), Europe.

FULIGINEUs, Fr., Hym. Eur., 525 (Mes.), Europe.

Fuliginosus, $F r$., Hym. Eur., 543 (Mer.), U. States, Europe.

Fuliao, $B . \& B r$. Linn. Joum. xiv. 53 (Res.), Ceylon.

FullaGeri, B., Linn. Journ. xvi. 54 (Placo.), Lord Howe Island.

Fulvitinctus, $R . \&$. C., Linn. Journ. x. 313 (Ino.), Cuba.

Fouvos (Scop.), Fr., Hym. Eur., 559 (Placo.), Australia, Ceylon, U. States,

Europe.

fulvus, Sihff., t. $262=$ pinicola, Fr.

royosus, Fr., Hym. Eur., 549 (Ano.), Venezuela, U. States, Cuba,

Europe.

FoNalIs, Fr., Epicr., 459 (Ano.), Guinea, India, Brazil.

ForCatus, Jungh., Fl. Jav. (Pleur.), Java.

Fuscatos, Fr., Hym. Eur., 569 (Ino.), Europe.

Foscidulus, Fr., Hym. Eur., 528 (Mes.), Europe.

fusco-albus, Jungh., Fl. Jara= Junghulinii, Lev.

fusco-badius, Pers, in Freyc. $=$ scabrosus, $F r$.

rosco-CaRneus, Pcrs., Myc. Eur. iii. 97 (Res.), U. States, Europe.

fusco-giluse, $\mathrm{Sch}$ w. = radiatus, $F r$. 
rl'sco-tutrsceis, Fckl, Sym. Myc., 18 (Res.), Europe.

fusco-purpureus (Pers.), Fr., Epic., $465=$ Trametes badia.

ruscus, Lev., Ann. Sci. Nat., 1846, 137 (Ino.), Java.

fuscus, Pers., Syn., $527=$ resinosus, Fr.

Galactinus, B., Hook. Journ., 1847, 321 (Ano.), U. States.

galbaxatus, B., Ann. Nat. Hist., 1843, 377 (Ino.), (country unknown).

Galeaensis, Mont., Syll., 158 (Placo.), Galega. gallicus, Fr., Sys. Myc., i. 345= Trametes gallica, Fr.

galirvaceds, $B$. \& Cke., Linn. Journ. xv. 379 (Pleur.), Brazil.

Gaudichaudi, Lev., Ann. Nat., 1844, 185 (Pleur.), Singapore.

Gayanus, Lev., Ann. Sci. Nat., 1846, 127 (Pleur.), Chili.

gelsoruM, Fr., Hym. Eur., 563 (Placo.), Europe.

gibberulosus, Lev., Ann. Sci. Nat., 1846, 129 (Ino.), Guiana. gibbosa, Whlbg., Ups. = borealis, $F r$.

arbmosus (Nees), Fr., Epic., 443, (Pleur.) Philippines, Java, Australia. giganteus, Fl., Dan., t. $1793=$ intybaceus, Fr.

giganteus, Harz., t. $32=$ squamosus, Fr.

giganteus, Fr., Hym. Eur., 540 (Mer.), U. States, Europe.

GIlvus (Schw.), Fr., Hym. Eur., 548 (Ano.), U. States, Mexico, Australia, Europe.

gilvus, Mont. = scruposus, $F r$.

gLabratus, Kalch., Hedwigia, xv. 114 (Pleur.), Australia.

glabrescens, B., Ann. Nat. Hist., 1839, 391 (Ino.), Mauritins.

glomeratus, Peck., 24th Report, N.Y. (Mer.), U. States. gonoporus, Jungh., fide Lev. = Dædalea sangrinea.

Gordonievsis (B. \& Br.), Fr., Hym. Eur., 579 (Res.), Europe, U. States ( ()

Gossypinus, Lev., Ann. Sci. Nat., 1843, 124 (Ino.), Europe.

Gourlisi, Berk., Fl. Tasm., 253 (Placo.), Tasmania

Gracilis, Berk., Ann. Nat. Hist., 1839, 384 (Mes.), India.

grammocephalus, $B$., Hook. Journ., 1842, 148 (Pleur.), Philippines, Admiralty Island, India, Cuba, Ceylon, Brazil, Indian Archipelago.

Gratus, $B$., Hook. Journ., 1852, 163 (Ino.), India.

GRaveolens (Schw.), Fr., Epic., 451 (Mer.), U. States.

GRIseus, Peck., 26th Report, 68 (Mes.), U. States.

GRYPH FFoRMIS, B., Hook. Journ., 1845, 54 (Placo.), Australia.

Guadelupensis, Lev., Ann. Sci. Nat., 1846, 134 (Ino.), Guadeloupe.

Golanensis, Mont., Syll., 153 (Mes.), Cayenne, Brazil.

GoxnII, Berk., Fl. Tasm., 253 (Ano.), Tasmania.

HÆDINUs, B., Hook. Journ., 1856, 234 (Ino.), Brazil.

hamatodes, Rostk., 4, t. $62=$ rufus, Fr.

Halesie, B. \& C., Grev. i. 52 (Ano.), U. States.

Hasskarlit, Lev., Ann. Sci. Nat., 1844, 190 (Ino.), Java, India, Brazil, Cuba.

Hasseltil, Lev., Ann. Sci. Nat., 1844, 187 (Ino.), Java.

Hadsmanni, Fr., Hym. Eur., 552 (Ano.), Europe.

Havasensis, B. \& C., Linn. Journ. x. 310 (Placo.), Cube.

Helveolds (Rostk.), Fr., Hym. Eur., 554 (Placo.), Europe.

helvolus, Fr., Nova Symb., 63 = Trametes.

hemiraphos, B., Hook. Joum., 1856, 193 (Pleur.), Brazil.

heMiCAPNoDEs, $B$. \& Br., Linn. Journ. xiv. 47 (Mes.), Ceylon.

hemileccos, B. \& C., Linn. Journ. x. 312 (Placo.), Cuba.

hemitrephiUs, Berk., Fl. N. Zeal., New Zealand.

Herbergii, Rostk., 29, t. 18 = spongia, Fr.

Heterochitcs (Bolt.), Fr., Hym. Eur., 544 (Mer.), Europe. 
heteroclitus, Sow. Fung., t. $367=$ ravidus, $F r$.

heteromorphus, Lev., Aun. Sci. Nat., 1846, 123 (Mes.), Brazil, Guiana. HETEROPORUs, Fr., Hym. Eur., 543 (Mer.), Europe.

heteroporos, Mont. Syll., 156 (PIaco.), Galegas.

hexagonoides, Fr. = Trametes.

HIAsis, Karst., Fungi Fenn. (Res.), Europe.

hibericus (B. \& Br.), Fr., Hym. Eur., 579 (Res.), Europe.

Hixwoleus, B. \& Cke., Linn. Journ. xv. 378 (Pleur.), Brazil.

HIRsutus, Fr., Hym. Eur., 567 (Ino.), India, Australia, Mexico, Java,

Siberia, N. Zealand, Fiji, Borneo, Brazil, Central America, U. States,

Tahiti, Cuba, Tasmania, Ceylon, Chili, Cape, Europe.

Hirsutulds, Schro., Amer. Bor., 373 (Ino.), U. States.

hirtelles, Fr., Nova Symb., 66 (Ino.), Mexico.

HIRTus (Quel.), Fr., Hym. Eur., 536 (Pleur.), Europe.

Hispidos, Fr., Hym. Eur., 551 (Ano.), Australia, O. States, Europe.

hispidus, Rostk., t. 31 =vulpinus, Fr.

nolsiexsis, Fr., Hym. Eur., 544 (Mer.), Europe.

HOLOLEUCU,, Kalch., Hedwigia, xv. 114 (Ino.), Australia.

holopheus, Mont., Syll., 163 (Ino.).

holotephnus, $B$. \& C., Linn. Journ. x. 315 (Ino.), Guiana, Cuba.

holoscleres, B., Hook. Journ., 1856, 200 (Placo.), Ceylon, St Domingo,

Brazil, Australia, Pegu.

hornodermus, Mont., Ann. Sci. Nat. v. 368 (Placo.), Brazil.

Hostmanni, Berk, Hook. Journ., 1842, 138 (Pleur.) Surinam.

Humboldtị, Pers., Myc. Eur. ii. 120=dubia.

Humisis, Peck., 26th Report, 69 (Pleur.), U. States.

HYALINUs, Berk., Fl. Tasm., 255 (Res.), Tasmania

HYBRIDUs, Sow., t. 289 (Res.), Europe.

HYDNICEPS, B. \& C., Linn. Journ. x. 305 (Mes.), Cuba.

hydnoides, Fr., El. i. $107=$ Trametes hydnoides, Fr.

HYDNOPHORUs, $B . \&$ \& Br, Linn. Journ. xiv. 55 (Res.), Ceylon.

hYDROPHILUs, $B$. \& $C$., Linn. Joum. x. 306 (Pleur.), Cuba.

HYPocITRINes, B., Linn. Journ. xv., 50 (Ano.) Bermuda.

BYPOCOCCINDS, B., Hook. Journ., 1847, 319 (Ano.), U. States.

hypoplastus, B., Hook. Journ., 1856, 174 (Mes.), Brazil, Pegu.

igniarius, Bolt = cinnamomeus, Fr.

igniarius, Fl., Dan., t. $953=$ pinicola, Fr.

igniariUs, Fr., Hym. Eur., 559 (Placo.), Australia, Ceylon, Brazil, India,

Philippines, Admiralty Island, Tasmania, Java, New Zealand,

U. States, Brit. N. America, Siberia, Europe.

IIICINColA, $B . \& C$., Grev. i. 52 (Ino.), U. States.

IMBERBIS (Bull), Fr., Hym. Eur., 543 (Mer.), Europe.

rMBbicatus, Fr., Hym. Eur., 542 (Mer.), Europe.

mpolitus, Fr., Nova Symb., 42 (Ano.), Costa Rica.

impuber, Sow., t. $195=$ gilvus, Schw.

mamcenus, Mont., Ann. Sci. Nat., 1842, 22 (Placo.), India.

incanus, Lev., Ann. Sci. Nat., 1846, 130 (Placo.), Australia.

INCARXATUS (A.\&S.), Fr., Hym. Eur., 573 (Res.), U. States, Europe.

incarnatus, Schum., Fl. Dan. =abietinus, Fr.

incexdiarius (Bong.), Fr., Hym. Eur., 517 (Mes.), Europe.

Incertus, Curr., Linn. Trans., 1876, 123 (Ano.), Pegu.

incertus, Pers., Myc. Eur., 337 = vaporarius, $F r$.

rxcostros, Fr., Epic., 437 (Ifes.), Guinea.

inconspicuds, Mig., Bull. Neerl., 1839, 454, Guiana.

incrassatus, B., Linn. Journ. xvi. 41 (Placo.), Cape York.

ivcrusi'ass, B. \& C., Grev. i. 54 (Res.), U. States. 
incrustans, Pers., Myc. Eur. i. $94=$ obliquus, Fr.

ixcrestatus, Fr., Nova Symb., 45 (Mes.), Costa Rica.

IXDECORus, Jungh., Fl. Jara, 51 (Ino.), Java.

inferxalis, Berk., Hook. Journ., 1843, 637 (Pleur.), Brazil.

inflexibiLIs, Berk., Hook. Journ., 1856, 192 (Placo.), Brazil.

informis, Cum., Act. Taur. = lobatus, Fr.

infundibuliformis, Pers., Syn., $526=$ melanopus, Fr.

infundibuliformis, Rostk., t. $27=$ Rostkovii, Fr.

ixquinatos, Lev., Ann. Sci. Nat., 1846, 140 (Ino.), India.

insularis, Pers., Myc. Eur. ii. 113=dubia.

Ixternos, Schw., Amer. Bor., 469 (Res.), U. States.

INTERCALARIS, $B$. \& Cke., Linn Journ. xv. 380 (Ano.), Brazil.

intermedius, Rostk. iv. t. 33 =arcularius, Fr.?

ixterruptus, $B$. \& Br., Linn. Journ. xiv. 55 (Res.), Ceylon.

Ixtoxsus, Berk., Flor. Tasm., 254 (Pleur.), Tasmania.

Ixtybaceus, Fr., Hym. Eur., 538 (Mer.), India, Europe.

intybaceus, B., =cichoraceus, $B$.

Ixzenges (Not.), Fr., Hym. Eur., 557 (Placo.), Europe.

Iodrvus, Mont., Syll., 167 (Ino.), Gniana, Brazil, Venezuela, Cuba.

IRIDIOIDEs, Berk., Fl. N. Zeal. (Ano.), New Zealand.

irpex, Schulz. in Fries = Schulzeri, Fr.

irregularis, Sow., t. $423=$ amorphus, Fr.

ISABELLinus, (Schw.), Fr., Epic., 475 (Ano.), U. States.

Isidiondes, B., Hook. Journ., 1843,.415 (Placo.), Ceylon, Cape, Australia, India.

JAPONICUS, Fr., Epicr., 442 (Pleur.), Japan.

Javanicus, Cke. (spadiceus, Jungh.) (Ino.), Java, Natal.

JTGIANDinds, Schw., Syn., 926 (Res.), U. States.

juglandis, Pers. Myc. Eur. ii. $53=$ squamosus, Fr.

juglandis, Schff. t. 101, $102=$ squamosus, $F r$.

juglandis, Schw., Syn. Car., 926= spissus, Fr.

JunghohnI, Fr., Nova Symb., 40 (Ano.), Java.

Kalchbrenneri, Fr., Hym. Eur., 531 (Mes.), Europe.

Kamphoveni, Fr., Nova Symb., 53 (Ano.), Tahiti.

Katui, Ehr., Hor. Phys., f. $12=$ xanthopus, Fr.

KeithII, B.\& Br., Ann. Nat. Hist. No. 1430 (Ano.), Europe.

Kerensis, Pass., Nuovo G. Bot. Ital. (Ino.), Abyssinia.

kermes, $B$. \& Br., Lin. Journ. xiv. 49 (Placo.), Ceylon.

KICKxIands, Lev., Ann. Sci. Nat., 1848, 122 (Ino.), Tropical America. Klotschii, B., Ann. Nat. Hist. iii. 383= Hexagona Klotschii, Fr.

Kanigir, B., Ann. Nat. Hist., 1843, 373 (Pleur.), Ceylon.

Korthalsir, Lev., Ann. Sci. Nat., 1846, 133 (Placo.), Java, Brazil, Guadeloupe, Sumatra.

k puatodes (Rostk.), Fr., Bym. Eur., 550 (Ano.), Europe.

laburni, Opiz. seznam, $136=$ dubia.

labyrinthicus, Mont. = Irpex maximus, $F r$.

labyrinthicus, Weinm., Ross, $313=$ Weinmanni, Fr.

LABRintricus (Schw.), Fr., Epic., 459 (Ano.), U. States.

laccatus, Pers., Myc. Eur. ii. $64=$ lucidus, $F r$.

laceratus, Curt., in Sillim. Journ.=pergamenus, Fr.

laceratus, B., Ann. Nat. Hist., 1839, 392 (Ino.), U. States, Cuba

LACERTS, Jungh., $F l$. Java (Pleur.), Java.

lacinatus (P.) Fr., Hym. Eur., 530 (Mes.), Europe.

lacrymans, Sauter, in Hedwigia, 1876, uncertain. 
uactecs, Fr., Hym. Eur., 546 (Ano.), Ceylon, U. States, Europe.

Laricolon, B., Linh. Journ. xvi. 46 (Amo.), Philippines.

lavigatus, Fr., Hym. Eur., 571 (Res.), Europe.

lavi, Pers. Myc. Eur, ii. $68=$ imberbis, Fr.

lanatus, Fr., Nova Symh., 74=Trametes lanata, Fr.

laneus, Pers. Myc. Eur. ii. 112 (monstrosa), (Res.), U. States, Europe.

Laxadidus, Fr., Epic., 442 (Pleur.), Guinea.

laricis, Jacq., Misc. t. $20=$ officinalis, Fr.

iate-Marainatus, Mont., Syll., 163 (Res.), Algetia.

LAtus, Berk., Ann. Nat. Hist. iii. 325 (Res.), Tasmania.

latus, Fr., Sys. Myc. i. $384=$ dubia.

LaurencIr, B., Fl. Tasm., 254 (Placo.), Tasmania.

LeIoderuUs, Mont., Syll., 168 (Ino.), Guiana.

LeNIs, Lev., Ann. Sci. Nat., 1848, 122 (Ino.), America.

LENTUS, Berk., Outl., p. 237 (Mes.), Brazil, U. States, Europe.

Lenziteus, Lev., Zoll. Verz, 17, Indian Archipelago.

LEONINUs (Klot.), Fr., Epic., 458 (Ano.), India, Guiana.

Leovotis, Kalch., Grev. iv. 73 (Placo.), Australia.

LEPIDEUS, Fr., Hym. Eur., 526 (Mes.), U. States, Europe.

LEPORINUS, Fr., Hym. Eur., 565 (Ino.), Europe.

LePriedrII, Mont., Syll., 155 (Pleur.), Guiana, New Ireland, Brazil.

LEPRODEs, (Rostk.). Fr., Hym. Eur., 535 (Pleur.), Europe.

LeProsus, Fr., Epic, 464 (Placo.), Brazil.

reptocephalUs (Jacq.), Fr., Hym. Eur., 528 (Mes.), U. States, Europe.

LEPTODERMUS, B. \& Br., Limn. Joum. xiv. 54 (Res.), Ceylon.

leptopus, Pers., in Freyc. Voy. $=$ umbraculum, Fr.

LeUcoloseds, Lev., Ann. Sci. Nat., 1846, 140 (Res.), U. States.

levcomallus, B. \& C., Linn. Jonirn. x. 308 (Ano.), Cuba.

ledcomelas (Pers.), Fr., Hym. Eur., 523 (Mes.), U. States, Europe.

Leucophads, Mont., Syll., 157 (Placo.), U. States.

teucoplaces, B., Fl. N. Zeal. (Res.), New Zealand.

leucoporus, Holms., Ot., t. $30=$ perennis, $F r$.

LEVEILlei, Cke. (cinerascens, Lev.) (Pleur.), Java.

Levissimus, Fr., Epicr, 470 (Placo.), Ceylon.

IIBOs, Berk., Linn. Journ. xiii. 163 (Ino.), Australia.

IICNoImes (Fr.), Mont., Syll., 166 (Placo.), India, Cuba, Brazil, Guiane, Ceylon, Jara.

Liebuanni, Fr., Nova Symb., 43 (Ino.), Mexico.

LIGNEUS, Berk., Ann. Nat. Hist., 1839, 387 (Placo.), St Vincents.

LIONosus (Klot.), Fr., Epicr., 471 (Placo.), India, Mauritius, Australia.

lignescens, Fr., Nova Symb., $42=$ lignosus, $K l$.

lilacinus, Schw., Sym. Car., 912= purpureus, Fr.

lilacinogilvus, Pers. = Feei, Fr.

Immbatus, Fr., Epicr., 479 (Ino.), Brazil, Australia

Limitatos, B. \& C., Grev. i. 50 (Res.), U. States.

Linduladir, Berk., Grev. i. 54 (Res.), U. States.

Lindmeimeri, B.\& C., Grev. i. 50 (Ano.), U. States.

Iineatus (Pers.), Fr., Epic., 477 (Ino.), Moluccas.

ungda (Nees), Fr., Epic., 443 (Pleur.), Java.

LINTEUS, B., U.S. Exp. (Ino.), Nicaragua.

imturarids, B. \& C., Sill. Journ., 1851, 94 (Ino.), Fiji.

cobatus (Huds.), Fr., Hym. Eur., 540 (Mer.), U. States, Europe.

zoвstus, Schw., Am. Bor., 409 (Placo.), U. States.

congIPEs, Lev., Ann. Sci. Nat., 1846, 125 (Pleur.), Guiane.

LONICERE, Weinm., Syll., 102 (Placo.), Europe.

loricatus, Pers., Myc. Eur. ii. $86=$ igniarius, Fr. (resupinate).

uvcidos, Fr., Hym. Eur., 536 (Pleur.), Cosmopolitan. 
Landii, Mont., Cuba, $393=$ Trametes rigida, Fr.

Livir, Fr., Epicr. 479 (Ino.), Brazil.

i.trules, B. \& U, Grev. i. 37 (Mes.), U. States.

L. TEonimides, B., Hook. Journ., 1856, 175 (Mes.), Brazil.

luteoporosus, Opiz., Lotus., 1855, 87-dubia.

I.crescens, Pers., Myc. Eur. ii. 71 (Ino.), Europe.

c.rec's (Nees), Fr., Epicr., 445 (Pleur.), Australia, Brazil, Cuba, Aru,

Nicobar, U. States, Sambulang.

3LACER, B., Hook. Journ., 1856, 176 (Pleur.), Brazil. macer, Somm., Lapp. = vaporarius, Fr.

araculatus, Peck., $26 t h$ Report, 69 (Mer.), U. State日.

Makraulos (Rostk.), Fr., Hym. Eur., 573 (Res.), Europe.

sacroporus, Lev., Ann. Sci. Nat., 1848, 132, Java.

macrotremus, Jungh. (fide Leveille) = Hexagona Molkenbœri, Lev.

3acllates, B., Hook. Journ., 1851, 80 (Mes.), India.

malacoderma, Fr. $=$ Trametes occidentalis, $F r$.

maxgifere, Lev., Ann. Sci. Nat., 1846, 129 (Placo.), Mahe.

Maxubriatus, Lev., Zoll. Verz., 17, Indian Archipelago.

sarasmioides, B., Hook. Journ., 1856, 175 (Mes.), Brazil.

Marchionicus, Lev., Ann. Nat., 1846, 300=Trametes.

Marginatus, Fr., Hym. Eur., 561 (Placo.), India, Arctic America,

U. States, Pegu, Europe.

marginatus, Pres., Syn., 534=pinicola, Fr.

Martius, B., Hook. Journ., 1856, 198 (Placo.), Brazil.

MAs'opord, Lev., Ann. Sci. Nat., 1844, 182 (Pleur.), Singapore.

maximus (Brot.), Fr., Hym. Eur., 529 (Mes.), Europe.

3iedulla-PaNis, Fr., Hym. Eur., 579 (Res.), U. States, Furope.

medullapanis, Secr., Myc., $108=0$ bducens, $F r$.

MEDUllaris, B., Hook. Journ., 1852, 140 (Placo.), India.

IEgaloma, Lev., Ann. Sci. Nat, 1846, 128 (Placo.), U. States.

segaloporus, Mont., Syll., 155 (Pleur.), Guiana.

MLgaloporus, Pers., Myc. Evr. ii. 88 (Res.), Europe.

Melaexus, Lev., Ann. Sci. Nat., 1846, 131 (Ino.), Java.

Melanoledcus, Lev., Ann. Sci. Nat., 1846, 141 (Res.), Bourbon.

melavoporus, Mont., Cuba, 422 (Placo.), Cuba.

MElaxopts, Fr., Hym. Eur., 534 (Pleur.), Mexico, U. States, Europe.

MELEAgRIS, $B$., Likn. Journ. xvi. 42 (Pleur.), Aru.

Mellets, $B . \&$ Br., Linn. Journ. xiv. 53 (Res.), Ceylon.

mellinus, Pers., Myc. Eur. ii. $96=$ sinuosus, Fr.

MeMbranacecs, Fr., Epicr., 481 (Ino.), Mexico, Cuba, Jamaica.

Mexaxdiaycs, Mont., Syll., 165 (Ino.), U. States.

IIexziesir, B., Linn. Journ. xiv. 46 (Pleur.), Ceylon, Sumatra. merismoides, Corda. = applanatus, $F r$, forma monstrosa.

serulixus, B., Fl. Tasm., 254 (Res.), Tasmania. mesentericus, Schff., t. $267=$ giganteus, $F r$.

Merexi, Klotsch., Nov. Act. N. Cur. xix. 236, Philippines.

MICANs (Ehr.), Fr., Hym. Eur., 573 (Res.), U. States, Europe.

Mrcheli1, Fr., Hym. Eur., 533 (Pleur.), Europe.

Microcyclus, Zipp., Ann. Sci. Nat., 1844, 188 (Ino.), Java.

Microloma, Lev., Ann. Sci. Nat., 1844, 183 (Mes.), Philippines. micromegas, Mont. = zonalis, $B$.

Microporcs, Fr., Epic., 472 (Placo.), Jamaica, U. States.

MICroscopices, Jungh., Fl. Jara (Ano.), Java.

sicrostoyes, B. \& C., Linn. Journ. x. 308 (Ano.), Cuba

Miniatus, Jungh., Fl. Java (Plear.), Java.

3risius, Fr., Hym. Eur., 536 (Pleur.), Europe. 
minimus, Jungh., 11. Java, 64, probably Laschia.

minimus, Rav., Grev. i. $65=$ elachistus, Berk.

Miquelii, Mont., I i n. Sci. Nat., 1845, 357=Hexagona Miquelii, Mont.

Murus, Kalch., Bulle. Mosc., 1877 (Placo.), Siberia.

Modestus (Kze.), Fr., Epic., 444 (Pleur.), Surinam, Java, Pegu.

modestus, Berk. = brunneolus, $\boldsymbol{F} r$.

mollis, A.\& S., $247=$ Weinmanni, Fr.

mollis, var. $\beta$, A. \& S., $247=$ fragilis, $F r$.

mollis, Rostk. ir. t. $25=$ erubescens, Fr.

mollis, Somm., Lapp., 271 = Trametes mollis, Fr.

MoluIs (Pers.), Fr., Hym. Eur., 547 (Ano.), U. States, Europe.

Mollidsculus, B., Hook. Journ., 1847, 320 (Ano.), U. States.

Molluscus, Fr., Hym. Eur., 578 (Res.), Chili, U. States, Europe.

monochrous, M. =languidus, Fr.

monsveneris, Jungh., Fl. Java, $61=$ leoninus, $F r$.

Montagnei, Fr., Hym. Eur., 530 (Mes.), Europe.

Moritzianus, Lev., Ann. Sci. Nat., 1846, $130=$ Trametes.

morosus, Kalch., Bot. Zeit. = benzoinus, Fr.

Mortuosus, Fr., Nova Symb., 48 (Placo.), Pulo.

MUCIDUs, Fr., Hym. Eur., 577 (Res.), U. States, Europe.

mucidus, Scop., Ann. = alligatus, Fr.

multicolor, Schœff. t. $269=$ zonatus, Fr.

Moltiformis, Mont., Syll., 156 (Pleur.), Guiana.

MUltuPlicatus, Mont., Syll., 156 (Placo.), Guiana.

murinus, Nees = fuscidulus, Fr.

murinus, Lev., Ann. Sci. Nat., 1844, 185= brunneolus, $B$.

murinus, Rost. iv. t. $57=$ subspadiceus, $F r$.

MURINUs, Kalch., Grev. iv. 72 (Ino.), Australia.

motabilis, B. \& C., Grev. i. 38 (Pleur.), Guiana, Cuba, Brazil, U. States.

MYCLODES, Kalch., Grev. iv. 75 (Mes.), Australia.

MYRRHINUS, Kickx., Bull. Brux., 1840 (Ino.), Brazil, Guiana, Cuba.

Naxus, Mont., Syll., 153 (Mes.), Algeria.

Naucinus, Fr., Nova Sym., 41 (Placo.), Mexico.

Nebulosus, B.\& C., Linn. Journ. x. 317 (Res.), Cuba.

NeEsIr, Fr., Hym. Eur., 564 (Placo.), Europe.

Nepalexsis, B., Hool. Journ., 1852, 162 (1no.), India.

Nephelodes, Lev., Aun. Sci. Nat., 1846, 125 (Pleur.), Australia, Brazil.

NePhRidios, B., Hook. Journ., 1856, 195 (Pleur.), Brazil, Cuba.

nidolans, Fr., Hym. Eur., 548 (Ano.), U. States, Europe.

nidulans, Secr. No. $47=$ lutescens, Fr.

Niger, B., Grev. i. 53 (Res.), Ceylon, U. States.

NigriCANS, Fr., Hym. Eur., 558 (Placo.), Cuba, Java, North America, U. States, Europe.

nigricans, Lasch., in Rabh. Exs. = versicolor, Fr.

NIGRIPES, Fr., Epicr., 435 (Mes.), Brazil.

nigripes, Wallr., Fl. Germ. iv. $598=$ picipes, $F r$.

nig Rocivctus, B., Ann. Nat. Hist., 1843, 377 (Ino.), Ceylon, India.

nigrosiargivatus, Schwe, $A m ., 375$ (Ino.), U. States.

nigropurpurascens, Schw., Am. Bor., $360=$ dichrous, $F r$.

NigropurPUREOs, Schw., Syn. Car., 925 (Res.), U. States.

Nilgherressis, Mont., Ann. Sci. Nat., 1842, 22 (Ino.), Cuba, India,

U. States, Centr. America, Brit. N. America.

nigrozonatus, Sauter, in Hedwigia, $1876=$ dubia.

NIPHODES, $B . \& B r$., Limn. Journ. xir. 55 (Res.), Ceylon.

Niskiensis, Pers., Myc. Eur. ii. $93=$ incarnatus, Fr.

nitens, Batsch., f. $225=$ lucidus, Fr. 
:iruxs, Fr., Epic., 463 (Placo.), Trop. America.

witilits, A. \& S., p. $258=$ amorphus, Fr.

Nitides, Fr., Hym. Eur., 574 (Res.), U. States, Europe.

sivecs, Jungh., Fl. Jav., 48 (Res.), Java.

xrvusus, B., Hook. Journ., 1856, 196 (Ano.), Cuba, Brazil.

xonIPEs, B., Hook. Joum., 1852, 136 (Mes.), India.

covelosus, Fr., Hym. Eur., 566 (Ino.), Europe.

Tordmanxi, Lev., Demid. Voy., (Res.), Europe.

xotopts, Lev., Ann. Sci. Nat., 1844, 194 (Pleur.), Java.

vove asglie, B. \& C., Grev. i. 51 (Placo.), U. States.

xcbilis, Fr., Epicr., 471_(Placo.), Guinea.

vececs, Fr., Nova Symb., 64 (Ino.), Costa Rica.

icmulularids, Fr., Hym. Eur., 536 (Pleur.), Europe.

nummularius, Schrad., p. 152=elegans, $F r$.

Nctaxs, Fr., Nov. Symb., 45 (Pleur.), Costa Rica.

onducens (P.), Fr., Hym. Eur., 577 (Res.), U. States, Europe.

obductus, B., Hook. Journ., 1845, 304 (Placo.), British America.

oblectaxs, B., Hook. Journ., 1845, 51 (Mes.), Brazil, Australia, Ceylon, India, Tasmania, New. Zealand.

obltecus, Berk. (fide Fries) = bulbipes, Fr.

obliquatus, Bull, t. $459=$ lucidus, $F r$.

oncrovus, Fr., Hym. Eur., 570 (Res.), Cuba, U. States, Ceylon, Australia,

Bermuda, Brazil, Europe.

onovates, Jungh, Fl. Java (Pleur.), Java.

onsoletus, Fr., Epic., 442 (Pleur.), Brazil.

ontesus, B., Ann. Nat. Hist., 1839, 390 (Ano.), British N. America.

oltusus, Pers., Obs.=igniarius, Fr.

obroltius, B. \& Cke., Grev. vii. (Placo.), U. States.

occidentalis, Kl., Ann. Nat. Hist., 1839, 393-Trametes occidentalis, Fr.

ocellates, B., Hook. Journ., 1856, 172 (Mes.), Brazil.

ochraceus, Pers., Syn., $539=$ zonatus, Fr.

ochrolaccatus, Mont., Ann. Sci. Nat., 1842 (Placo.), Philippines.

ochroledces, B., Hook. Journ. iv. 52 (Ano.), Australia, Madagascar,

Tasmania.

ochrotinctus, B. \& O., Proc. Amer. Acad. iv. 122 (Pleur.), Japan, Cuba, Bonin Isles.

odoratus, Fr., Sys. Myc. j. 373=Trametes.

(Erstedti, Fr., Nova Symb., 47 (Placo.), W. Indies.

officixalis, Fr., Hym. Eur., 555 (Placo.), Europe.

olwaceofuscus, B., Linn. Journ. xiv. 46 (Mes.), Ceylon.

omalophilus, Mont., Cub., 423 (Placo.), Cuba, Guiana, Brazil.

oiphalodes, B., Hook. Journ., 1866, 172 (Mes.), Brazil.

opaces, M. \& B., Mont., Syll., 161 (Pleur.), Brazil.

orbicularis, B., Ann. Nat. Hist. iii. 324 (Res.), Tasmania.

orbicularis, Sauter, in Hedwigia, 1876.

onbiforuis, Fr.. Epicr., 463 (Placo.), Guinea, Australia.

ossecs (Kalch.), Fr., Hym. Eur., 541 (Mer.), Europe.

ostrea, Pers., Myc. Eur. ii 119.

ostreeforMis, B., Linn. Journ. xvi 46 (Ano.), Philippines.

ostreatcs, Lev., Ann. Sci. Nat., 1816, 128 (Pleur.), Brazil.

orixcs, Fr., Hym. Eur., 523 (Mes.), U. States, Europe.

oxrbatcs, B. \& C., Linn. Journ. x. 317 (Res.), U. States, Cuba.

oryporus, Sauter, in Hedwigia, 1876.

aronioides, B., Hook. Journ., 1851, 83=Trametes.

PAChypes, Mont., Syll., 154 (Pleur.), Cuba.

TRANS. HOT. SOc. VOL. XIII. 
pachypus, Pers. Myc. Eur. ii. $47=$ politus, $F r$. pachyus, Rostk., 27, t. $5=$ contiguus, Fr.?

P.Ecilus, Berk. ( $=$ petalodes, B.) (Placo.), Brazil.

Pala, Lev., Ann. Sci. Nat., 1844, 183 (Pleur.), Surinam. paleaceus, Fr., Epic., $471=$ Trametes.

Pallescens, Fr., Hym. Eur., 546 (Ano.), U. States, Europe. pallcscens, Schrad. p. $134=$ leptocephalus, Fr.

Pallidocervinus, Schw., Syn., 166 (Ino.), U. States, Cuba.

Pallidus, B., Hook. Journ., 1856, 176 (Pleur.), Brazil.

Pallidus (Schulz.), Fr., Hym. Eur., 533 (Pleur.), Europe. palmatus, Sauter in Hedwigia, 1876.

palmicola, B. \& C., Linn. Journ. x. 317 (Res.), Cuba.

Palustris, B. \& C., Grev. i. 51 (Placo.), U. States.

PaNdani, Fr., Epicr., 469 (Placo.), Cochin China.

Paxsus, B., Hook. Journ., 1856, 169 (Mes.), Brazil.

papyraceus, Fr., Epicr., 481 (Ino.), Costa Rica.

Paprraceus, Schw., Amer. Bor., 442 (Res.), U. States.

PaRadoxus, Fr., Hym. Eur., 555 (Placo.), Europe.

Parilis, Fr., Pl. Preiss., 136 (Res.) Australia.

paruula, B., Hook. Jourm., 1856, 173 (Mes.), Brazil.

Partitus, B., Hook. Journ., 1856, 170 (Mfes.), Brazil.

parvulus (Klot.), Fr., Epicr, 435 (Mes.), N. America.

PARVulus, Schw., Am. Bor., 386 (Ino.), U. States.

Passerinus, B., Hook. Journ., 1856, 175 (Pleur.), Brazil.

Padletil, Fr., Hym. Eur., 541 (Mier.), Europe.

pavonius (Hook.), Fr., Epicr., 477 (Ino.), Magdalena, New Granada.

Peckianus, Cke. (favidus, Peck.) (Mes.), U. States.

PeCtinatus (Klot.), Fr., Hym. Eur., 559 (Placo.), India, Cuba, Philippines,

Europe.

PECTUNCOLUs, Lev., Ann. Sci. Nat., 1846, 138 (Ino.), Cuba, India.

PEDIFORMIS, Fr., Epicr., 463 (Placo.), Guinea.

Pequanus, Mont., Syll., 158 (Placo.), Pegu.

pelleporus, Bull., t. 501, f. $2=$ adustus, Fr.

pelleporus, Secr., Myc. = dichrous, Fr.

pelleporus, Sow., t. 230=pallescens, $F r$.

PEllicula, Jungh., Fl. Java, 44 (Res.), Java.

Pelliculosus, B., Hook. Journ. vi. 575 (Auo.), Tasmania. pellitus, Meyer, Fl. Esseq., $304=$ Trametes fibrosa, Fr. pellucidus, Fr., Sys. Myc. i. 352.

penetralis, Smith, Journ. Bot., 1875, 98 (Pleur.), Europe.

Peltates, Fr., Nova Symb., 36 (Pleur.), Costa Rica

Peradenie, B. \& Br., Linn. Journ. xiv. 51 (Ino.), Ceylon.

Perennis, Fr., Hym. Eur., 531 (Mes.), U. States, Penang, Europe.

PeRGa MiENus, Fr., Epic., 480 (Ino.), U. States, Mexico.

PERonatus (Schulz.), Fr., Hym. Eur., 532 (Mes.), Europe.

PERoxydatus, B., Linn. Journ. xvi. 38 (Pleur.), Australia.

PERPUsillus, Pers., Ann. Sci. Nat., 1844, 191 (Placo.), N. America.

PERsicinus, $B . \&$ \&., Grev. i. 37 (Mes.), U. Stater.

personatus, $B$. \& Br., Linn. Journ. xiv. 51 (Ino.), Ceylon.

Persooxir, Fr. (Placo.), Australia, Ceylon, Bermuda, Moluccas, Pegu,

Admiralty Island.

pertusus, Pers., Myc. Eur. ii. 103=corticola, Fr.

PERULA, Fr., Epicr., 437 (Mes.), Brazil, Guiana.

PES-CAPRe (Pcrs.), Fr., Hym. Eur., 524 (Mes.), Europe.

pes-simie, B., Hook. Journ., 1589 (Pleur.), Brazil.

Petaliformis, B. \& C., Linn. Journ. x. 307 (Pleur.), Cuba.

petalodes, B., Hook. Journ., 1856, $198=$ pæcilus, $B$ 
peraloinfa, Fr., Hym. Eur., 536 (Pleur.), Europe.

l.H. Wurts, Mont., Syll., 165 (Ino.), Cayenne.

fHxaduls, Mont., Syll., 154 (Mes.), Columbia.

l'tels, Lev., Ain. Sci. Nat, 1846, 132 (Ino.), Java, Ceylon.

l'uninpusesists, Berk., Hook. Journ., 1842, 148 (Pleur.), Philippines.

imbmopioncs, Berk., Fl. N. Zeal. (Pleur.), New Zealand.

phoclses, B. \& Br., Linn. Journ. xiv. 52 (Ino.), Ceylon.

Wiles, Fr, Hym. Eur., 534 (Pleur.), Ceylon, Brazil, U. States, Cuba,

India, Australia, Europe.

pletilis, B., Hook. Journ., 1852, 162 (Ino.), India.

nictes (Schulz.), Fr., Hym. Eur., 531 (Mes.), Europe.

fll.coponcs, Mont., Syll., 163 (Ino.), Cayenne.

ilot.e, Silw., Am. Bor., 370 (Ano.), U. States.

pini, Pers, Myc. Eur. ii. 83=Trametes pini, Fr.

rixicola, Fr., Hym. Eur., 561 (Placo.), India, Cuba, U. States, Siberia,

Europe.

pisi Caxanexsis, Schw., Am. Bor., 410 (Placo.), U. States.

risirus, Fr., Epicr., 479 (Ino.), Guadeloupe, U. States, Cuba, British

America, St Thomas, Central America, India, Pegu.

pithyus, Chaill. = borealis, $F r$.

rlacketa, Fr., Hym. Eur., 572 (Res.), Europe.

Pi.Acodes, Kalch., Grev. iv. 73 (Placo.), Australia.

Placores, Lev., Ann. Sii. Nat., 1846, 124 (Pleur.), Java.

planus, Wallr., Fl. Germ., ii. 602.

platrpilus, Lev., Ann. Sci. Nat., 1844, 192 (Ino.), Java.

p'latrponcs, Berk., Hook. Journ., 1851, 81 (Pleur.), India. platyporus, Pers., Syn., 521 = squamosus, $F r$.

plebeICs, Berk., Fl. N. Zeal. (Ino.), India, N. Zealand, Brazil, Cuba

Plicatos, Blume, Ann. Sci. Nat., 1844, 185 (Pleur.), Java.

plicatus, Pers., Myc. Eur. ii. $212=$ salicinus, Fr.

rlisbes, Lev., Ann. Sci. Nat., 1846, 136 (Ino.), Guadeloupe.

I'tcsibosus, Fr., Nova Symb., 77 (Ino.), Mexico.

pocss, B., Linn. Journ. xvi. 51 (Ino.), Japan.

piciles, B., Ann. Nat. Hist., 1843, 372 (Pleur.), Ceylon ?

rolites, Fr, Hym. Eur., 625 (Mres.), Europe.

polycephalus, Pers., Syn., $519=$ umbellatus, Fr.

rolydactrlos, $B$., Hook. Journ., 1856, 196 (Mer.), Brazil.

roligramues, $B$. \& C., Linn. Journ. \& 307 (Pleur.), Cuba

polygrammus, IIont., Cuba, $379=$ Hexagona polygramma, Mont.

polymorphus, Hoffm. = Trametes odorata, $\mathrm{Fr}$.

rolTionphus, Rostk., 4, t. 56 (Ino.), Europe.

polyporus, Bull, t. $469=$ fuligineus, $F r$.

polystictus, Pers., Myc. Eur. iii., uncertain.

rolytropus, $B$. \& Br., Linn. Journ. xiv. 49 (Placo.), Ceylon.

rolrzonds ( $P$.), Fr., Epic., 477 (Ino.), Borneo, Guadeloupe, Martinique. jomaceus, Pers. =igniarius, Fr.

Popclives, Fr., Hym. Eur., 546 (Placo.), U. States, Europe.

populinus, Schulz. = vulpinus, Fr.

jopulneus, Poll., Pl. Ver., $34=$ castaneus, Secr.

PORIPEs (Schw.), Fr., Nova Symb., 32 (Pleur.), U. States,

porphyrites, B., Hook. Journ., 1856, 433 (Pleur.), Brazil, Cube.

rortestoscs, B., Hook. Joum., 1844 (Placo.), Australia, Japan, Tasmania.

rortoricexsis, Fr., Epic., 483 (Res.), Porto Rico.

proboscideus, Jungh. (fide Lev.) = notopus, Lev.

PRocercs, B., Hook. Journ., 1856, 171 (Mes.), Brazil.

Prolificdxs, Fr., Epic., 143 (Pleur.), Mexico, U. States.

proteus, B., Hook. Journ., 1843, $414=$ Trametes. 
prcinatus (Klot.) Fr., Epic., 473 (Ino.), Cuba, Mauritius. pseudoboletus, Jacq., Austr. = lucidus, Fr. pseudoigniarius, Bull, t. $458=$ dryadeus, $F r$.

psiloderius, B. \& $M$., Mont. Syll., 167 (Ino.), Brazil.

PtergGodes, Fr., Epic., 445 (Pleur.), Guinea.

PUdescexs, Fr., Hym. Eur., 553 (Ano.), U. States, Europe.

PUdens, B., Hook. Journ., 1852, 138 (Pleur.), India.

PUlChellus, Schw., Amer. Bor., 426 (Res.), U. States.

PULCHER, Fr., Epic., 437 (Mes.), Guinea.

Pollus, B. \& M., Mont., Syll., 157 (Placo.), Java.

pulvinatus, Whlbg., Suec. = spumeus, $\boldsymbol{F r}$.

ponctates, Fr., Hym. Eur., 572 (Res.), Europe.

pUnctatus, Jungh., Fl. Java, 64 (Placo.), Java.

purgans, Pers., Syn., 531 = ofticinalis, Fr.

purporascens (Hook.), Fr., Epic., 473 (Ino.), Rio Magdalena.

purpureus, Rostk., 27, t. $3=$ violaceus, $F r$.

PURPUREUS, Fr., Hym. Eur., 572 (Res.), U. States, Europe.

pusillus, Schrad. = bramalis, Fr.

pUstulosus, Zoll., Arch. Neerl. (Ino.), Java.

PUtidus, Fr., Nova Symb., 35 (Pleur.), Costa Rica.

PYRRhoporus, Mont., Syll., 162 (Res.), Algeria.

QUERcinds (Schrad.), Fr., Hym. Eur., 555 (Placo.), Europe.

racodioides, Pers., Myc. Eur. ii. 113= bombycinus, Fr.?

Radiatus, Fr., Hym. Eur., 565 (Ino.), Cuba, U. States, Venezucla, Europe.

radiato-rogosus, Berk., Ann. Nat. Hist. iii. 323 (Mer,), Tasmania.

Radicatus, Schw., Amer. Bor., 331 (Mes.), U. States.

RanUla, Fr., Hym. Eur., 578 (Res.), U. States, Europe. ramosissimus, Secr., Myc. = intybaceus, Fr.

ramosissimus, Scop. = umbellatus, $F r$.

ramosus, Bull, t. $418=$ imbricatus, Fr.

RASIPES, B., Linn. Journ. xvi. 49 (Pleur.), Admiralty Island.

RaVENaLE, B. \& Br., Linn. Journ. xiv. 53 (Res.), Ceylon.

Ravenalii, B. \& C., Grev. i. $38=$ cretatus, $B$.

RavenaliI (B.\&Fr.), Fr., Nova Symb., 66 (Res.), U. States.

Ravidos, Fr., Hym. Eur., 566 (Ino.), Europe.

RENatcs, B., Hook. Journ., 1856, 170 (Mes.), Brazil.

Renvyi, B. \& Br., Ann. Nat. Hist. No. 1433 (Res.), Europe.

REsinosus, Fr., Hym. Eur., 554 (Placo.), U. States, Europe. resinosus, Rostk., 4, t. $29=$ Trametes radiciperda, Hart.

resupinatus, Bolt., t. $165=$ spongiosus, $F r$.

resupinatus, Sow., t. $424=$ Dædalea vermicularis, $P$.

Reticulatus, Fr., Hym. Eur., 580 (Res.), U. States, Europe.

rhabarbarinus, B., Ann. Nat. Hist., 1839, 388= senex, $N$.

R日eades, Pers., Myc. Eur. ii. 69 (Ano.), Europe.

RHEICOLOR, B. \& C., Linn. Journ. x. 303 (Ino.), Cuba.

nhinocephalus, Berk., Fl. Tasm. (Ano.), Tasmania.

RHINocerrus, ${ }^{*}$ Cooke, in Herb. (Mes.), Penang.

RHIPIDIOs, B., Hook. Journ., 1847, 319 (Pleur.), Ceylon, Brazil, Guiana,

Cuba, U. States, Australia.

RHIZOMORPHA, Mont., Syll., 161 (Mes.), Guiana.

RHODELlus, Fr., Hym. Eur., 573 (Res.), U. States, Europe.

* Pileus, 7-9 inches broad, striate, zoned, not $\frac{1}{4}$ inch thick ; stem 8 inches long, $\frac{1}{3}$ inch thick, laccate; proceeding from a hard sclerotioid base, 3 inches in diameter; pores minute, round, regular. 
riodonendri, Schw., Am. Bor., 436 (Res.), U. States. modornaus, Lev., Ann. Sci. Nat., 1844, 190 (Placo.), Java. thombiporus, Pers., Myc. Eur. ii. 211 = arcularius, $F r$. nurriphleus, Mont., Ann. Sci. Nat. v. 369 (Placo.), Brazil. ribesius, Pers., Myc. Eur. ii. $80=$ ribis, Fr.

mивs, Fr., Hym. Eur., 560 (Placo.), U. States, Europe. ricides, Lev., Ann. Sci. Nat. 1844, 189 (Ino.), Java. risosus, B., Hook. Journ., 1845, 54 (Placo.), Australia, Cape. hivelosos, B. \& C., Linn. Journ. x. 318 (Res.), Cuba. noblrieos, Fr., Hym. Eur., 557 (Placo.), Europe. rosamala, Jungh. (fide Lev.) = rhodophæus, Lev. rosarum, Weinm., Ross., $319=$ candidus, Fr. Reseonlbus, Jung., Fl. Java, 43 (Res.), Java. rosenporis, Rostk. 27, t. $12=$ amorphus, $\mathrm{Fr}$.

noseus (A. \& S.), Fr., Hym. Eur., 562 (Placo.), Europe. Rostkovir, Fr., Hym. Eur., 534 (Pleur.), Europe. rubella, Pers., Obs. i. $14=$ mollis, Fr. ruber, Pers. Myc. Eur. ii. 43. rubescens, A. \& S., t. 11, f. $2=$ Trametes rubescens, $F r$. rebidus, B., Hook. Journ. xiv. 49 (Ano.), Ceylon, Pegu. rubiginosus, Schrad., Spic., $168=$ resinosus, Fr. rubiginosus, B., Ann. Nat. Hist. iii. $324=$ Laurencii, $B$. ruluiginosus, Rostk. iii. to 32.

nubricus, B., Hook. Journ., 1851, 81 (Mer.), India. lLBRIPES, Rostk., 28, t. 16 (Mes.), Europe. rudis, Lev., Ann. Sci. Nat., 1846, 133=subfulvus, Cke. nedis, Berk., Ann. Nat. Hist. iii. 1839, 323, Tasmania. nupescens, Fr., Hym. Eur., 529 (Mes.), U. States, Japan, India. rufescens, Rostk., 4, t. 7 =tomentosus, $F r$. nefontratus, B., Hook. Journ., 1856, 174 (Mes.), Brazil. refoflavos, B. \& C., Linn. Journ. x. 310 (Placo.), Cuba, Ventuela. livfopallidus (Trog.), Fr., Hym. Eur., 561 (Placo.), Europe. nuFes (Schrad.), Fr., Hym. Eur., 573 (Res.), Europe.

rugosus (Nees), Fr., Epic., 437 (Mes.), Ceylon, Australia, India, Guinea, Java. rugosus, Sow. t. $422=$ alligatus, $F r$.

rugosus, Trog., Schw., $401=$ corrugis, $F r$.

rugclosus, Lev., Ann. Sci. Nat., 1814, 188 (Placo.), Java.

rugclosus, Lasch., Rabh. F. Eur., 16 (Ino.), Europe (an P. velutinus, Fr., var. ?)

Russiceps, B. \& Br., Linn. Journ. xiv. 48 (Pleur.), Ceylon. Missograme, B., Linn. Joum. xvi. 45 (Ino.), Moluccas. nutilass, Fr., Hym. Eur., 548 (Ano.), U. States, Europe. rutilans, Rostk., t. $36=$ testaceus, $F r$.

nutrosus (Rostk.), Fr., Hym. Eur., 525 (Mes.), Europe.

S.lccardoi, Che. (sericellus, Sacc.), (Mes.), Europe. sacer, Fr., Epic., 436 (Mes.), Guinea, Cape Java. Sagreanus, Mont., Cuba, t. xvi. f. 4=Trametes. salebrosus, Lasch., Rabh. Herb. Myc., 1666=nodulosus, Fr. sulicinus, Bull, t. $433 \mathrm{f}$. $1=$ albus, $F r$.

s.l.icines, Fr., Hym. Eur., 560 (Placo.), U. States, Australią, New Zealand, Cape, Europe.

sıi.igce, Fr., Hym. Eur., 544 (Mer.), Europe.

ill.sionicolor, B. \& C. Grev. i. 53 (Res.), U. States.

S.M. 1 I. B. \& C., Grev. i. 54 (Res.), U. States. 
eavatinarius (Klot.), Fr., Epic., 471 (Placo.), Mauritius, Indian Archipelago.

sanaurveds, Fr., Epic., 444 (Pleur.), India, Ceylon, Penang, Hawaii, Guinea, Mexico, Australia, Mauritius, Surinam, Java, Hong Kong, Cape, Brazil, Philippines, Sumatra, Singapore, Borneo, New Zealand, Tasmania, Cuba, United States, West Indies, S. Africa.

sanauinolentus, Fr., Hym. Eur., 578 (Res.). U. States, Europe.

sarcitus, Fr., Nova Symb., 50 (Placo.), W. Indies.

SaRTwelli, B. \& C., Grev. i. 51 (Ino.), U. States.

sassafras, Schw., Amer. Bor., 437 (Res.), U. States.

BCA BRICEPS, B.\& C., Linn. Journ. x. 305 (Mes.), Cuba.

bCabrosds (P.), Fr., Epic., 469 (Placo.), Mauritius.

BCALARIS, B., Hook. Journ. viii. 236 (Placo.), Brazil.

scalaris, Pers., Myc. Eur. ii. 90= Trametes serialis, Fr.

scanicus, Fr., Hym. Eur., 549 (Ano.), Europe.

scansilis, B., Linn. Journ. xvi. 53 (Placo.), Tahiti.

scarrosus, B. \& C., Grev. i. 52 (Ino.), U. States.

SchosiburackII, M. \& B., Hook. Journ., 1844, 331 )Mes.), Guiana.

Scholzeri, Fr., Hym. Eur., 556 (Placo.), Europe.

Scaweinitzir, Fr., Hym. Eur., 529 (Mes.), U. States, India, Europe.

sCleroderises, Lev., Ann. Sci. Nat., 1846, 129 (Placo.), Guadeloupe.

sClerodes, B., Linn. Journ. x. 311 (Placo.) Cuba.

sclerontrees, B. \& C., Linn. Journ. x. 312 (Placo.), Cuba.

sCleropodrus, Lev., Ann. Sci. Nat., 1846, 123) Mes.), Bourbon.

scobinaceus, Cum. Act. Taur. = pescapræ, Fr.

Scopolii, Pers., Myc. Eur. ii. 120, uncertain:

scopulosus, B., Hook. Jour., 1852, 143 (Placo.), Indie.

scorteds, Fr., Nova Symb., 73 (Ino.), Pulo Milu.

scoticus, Klot. =annosus, $F r$.

scruposus, Fr., Epic., 473 (Ino.), India, Tasmania, New Zealand, Mexico, Cuba, U. States, Australia, Brit. N. America, Aru, Pegu. scutatus, Pers:, Myc. Eur. ii 85, uncertain.

sCUTELlates, Schw., Am. Bor., 387 (Ino.), U. States.

scutiger, Kalch., Enum. = Kalchbrenneri, $\boldsymbol{F r}$.

scutiger, Fr., Elen. i. $73=$ - Hexagona scutigera, Fr.

scrtinus, B., Ann. Nat. Hist., 1843, 376 (Ino.), Philippines. sebaceus, Leyss., Hal. = destructor, Fr.

SeCERnibilis, B., Hook. Journ., 1847, 500 (Ano.), Ceylon, Guinea. sector (Ehr.), Fr., Epic., 480 (Ino.), Tasmania, Brazil, Cuba, Guiana. selectus, Karst. Not. Fenn. = flavus, Karst.

semiclatsus, B., Hook. Journ., 1856, 193 (Pleur.), Brazil.

semidiaitaliformis, $B$, Linn. Journ. xvi. 39 (Ano.), Australia.

seminigrita, B. \& Cke., Linn. Journ. xv. 377 (Pleur.), Brazil.

semiovatus, Schœff., t. $270=$ pinicola, Fr.

semipellucides, Zool., Arch. Neerl. (Ino.), Java. semipatera, Pers. =lucidus, $F r$.

semisupinus, $B$. \& $C$., Grev. i. 50 (Ano.), U. States.

semitostus, B., Hook. Joum., 1852, 143, (Placo.), India.

senex (Nees), Mont., Syll, 160 (Placo.), India, Cuba, Juan Fernandez,

Guiana, Brazil, Ceylon, Venezuela, Aru, Philippines, U. States.

serialis, Fr., Sys. Afyc. i. $370=$ Trametes.

serialis, Rostk. t. $49=$ zonatus, Fr.

sericellus, Sacc., Myc. Veneti., $818=$ Saccardoi, Cke.

sericeldUs, Lev., Ann. Sci. Nat., 1846, 125 (Pleur.), Ceylon.

sericeohirsutus, Klot., Linn. viii. $483=$ Hexagona sericea, $F r$.

serpentarius, Pers., Myc. Eur. ii. $82=$ annosus, Fr.

seTIPORts, B., Hook. Journ., 1847, 505 (Ino.), Ceylon, Brazil. 
siderotdes, Lev., Ann. Sci. Nat., 1844, 182 (Pleur.), Sumatra, Java. sIMItIs, B., Hook. Joum., 1843, 635 (Mes.), Brazil, Cuba.

sinensis, Fr., Sys. Myc. i. $345=$ Trametes.

sinuosus, Fr., Hym. Eur., 576 = Trametes.

sistotrema, A. \& S., $243=$ Schweinitzii, Fr.

sobriUs, B. \& C., Linn. Journ. x. 316 (Ino.), Cuba.

soloniensis, Fr., Hym. Eur., 553 (Placo.), Europe.

sorbICOLA, Fr., Hym. Eur., 570 (Res.), Europe.

sondidulos (B.), Fr., Nova Symb., 64 (sub sordidus), (Placo.), Costa Rica. sordidcs, Lev., Ann. Sci. Nat., 1844, 192 (Placo.), America, Guadeloupe. sordulentus, Mont., Syll., 160 (Placo.), Chili.

spadiceus, Jungh., Fl. Java, $54=$ Javanicus, $C$.

spadiceds, Berk, Ann. Nat. Hist., 1839 (Placo.), India.

spatholatus (Hook.), Fr., Epic., 443 (Pleur.), Central America, Peru.

spectabilis, Fr., Nova Symb., 32 (Mes.), U. States.

sPIssus (Schw.), Fr., Epic., 465 (Placo.), U. States.

sPlendens, Lev., Ann. Sci. Nat., 1844, 187 (Ino.), Java.

SPLendens, Peck., 26th Report, 68 (MIes.), U. States. = perennis, Fr. var. 1

Splitgerneri, Mont., Syll, 102 (Ino.), Surinam, Mexico, Cuba, Pegu.

SPoNGia, Fr., Hym. Eur., 542 (Mer.), Europe.

spongiosts, Fr., Sys. Myc. i. 377 (Ano.), U. States, Europe.

Spraquei, B. \&. C., Grev. i. 50 (Ano.), U. States.

spumeus, Fl., Dan. t. $1794=$ epileucus, Fr.

spomeus (Sow.), Fr., Hym. Eur., 552 (Ano.), U. States, Europe.

spurces, Lev., Ann. Sci. Nat., 1846, 135 (Ino.), Guadeloupe.

squalidUs, Fr., Epic., 469 (Placo.), Brazil.

sqUaMafoRMis, B., Hook. Journ., 1852, 139 (Pleur.), India.

squamosus, Fr., Hym. Eur., 682 (Pleur.), India, Europe.

stalactites, Hoffm., Veg. Crypt. = roseus, Fr.

Steinheiliands, B. \& Lev., Ann. Sci. Nat. (Ino.), Martinique.

Stephensii (B. \& Br.), Cooke, Hdbk. = Trametes.

stereinus, B. \& C., Linn. Jour. x. 308 (Pleur.), Cuba, Brazil, Admiralty

Islands

StEREOIDES, Fr., Hym. Eur., 569 (Ino.), U. States, Europe.

Stevenir, Lev., in Demidoff. Voy. (Placo.), Siberia, Europe.

stipitarids, B. \& C., Linn. Journ. x. 304 (Mes.), Brazil, Cuba.

stiptices, Fr., Hym. Eur., 546 (Ano.), U. States, Europe.

striatus, Fr., Epic., 480 (Ino.), Peru.

strigates, B., Hook. Journ., 1847, 502 (Ino.), Ceylon.

strigosus, Schulz. = vulpinus, $F r$.

strobiliforuis, Fr., Sys. Myc. i. 352.

struMostr, Fr., Epic., 462 (Placo.), Guinea.

subcinereus, B. Ann' Nat. Hist., 1839, 391 = adustus, Fr.

sUBERIs, Mont. Syll:, 162 (Res.), Algeria.

suberosus, Batsch. El., f. $226=$ adustus, Fr.

suberosus, Bolt., t. $162=$ Trametes odora, Fr.

suberosus, Bull, t. $482=$ nidulans, Fr.

suberosus, Linn., Suec. = betulinus, Fr.

suberosus, Sow., t. $288=$ cytisinus, $B$.

suberosus, Whlb., Ups. = spumeus, $F r$.

suberosus, Kromb., t. 48, f. 11-14=quercinus, Fr.

subfuavus, Lev. Ann. Sci. Nat., 1846, 500, U. States.

sebflexibilis, B. \& C., Linn. Journ. x. 311 (Placo.), Cuba.

sDBFULvUs, Cke. (rudis., Lev.), Ino.), Guadeloupe.

SUBF USCUS FlaVIDUs, Rostk., 27, t. 11, (Res.), Europe.

subaelativosus, $B$. \& Br., Ann. Nat. Hist. No. 1569 (Res.), Europe.

subaiganteus, B. \& C., Grev. i. 49 (Mer.), U: States. 
semliberates, $B$. \& C., Linn. Joum. x. 318 (Res.), Cuba. submembranaceus, Santer, in Hedwigia, 1876.

strolivacets, $B$. \& C., Linn. Journ. x. 313 (Ino.), Cuba.

subpertucides, B., Linn. Joum. xvi. 51 (Ino.), Japan.

subpileatus, Weinm. 332 =annosus, $F r$.

subpulverulextus, $B . \&$ C., Linn. Joum. x. 306 (Pleur.), Cuba.

subsPadicecs, Fr., Hym. Eur., 570 (Res.), U. States, Europe.

subsquajiosus, Fr., Hym. Eur., 523 (Mes.), U. States, Europe. subsquamosus, Secr. = cristatus, Fr.

substrictus (Rostk.), Fr., Hym. Eur., 582 (Mes.), Europe.

substrict us, Bolt., t. $270=$ fuscidulus, Fr.

soBstuppeds, B. \&. Cke., Linn. Journ. xv. 381 (Ano.), Brazil. subtomentosus, Bolt., t. $87=$ perennis, Fr.

subvinctes, $B$. \& Br., Linn. fourn. xiv. 53 (Res.), Ceylon.

sUlCIFER, B. \& Cke., Linn. Journ. xv. 383 (Ino.), Brazil.

solforatus, Fr., Nova s'ymb., 63 (Ino.), Mexico.

sULFURECs, Fr., Hym. Eur., 542 (Mer.), India, U. States, Tasmania, Cuba,

Ceylon, Europe.

Sullivanti, Mont., Syll., 163 (Ino.), U. States.

suPerficialis, Schw., Syn. Car., 924 (Res.), U. States.

superposirts, Berk., Linn. Journ. xiii. 161 (Pleur.), Australia.

supinus, Fr., Epicr., 471 (Placo.), Jamaica, N. America.

Sorinamensis, Miq., Bull. Neerl., 1839, 454 (Placo.), Surinam. sutorius, Scop. - betulinus, Fr.

Swartzianus, Lev., Ann. Sci. Nat., 1846, 132 (Ino.), Jamaica.

8ymphyton (Schw.). Fr., Epicr., 460 (Ano.), U. States, Mexico.

Tabacinos, Mont., Syll., 167 (Ino.), Aru, Chili, Juan Fermandez, Mauritius, N. Zealand.

tabulaformis, B., Hook. Journ., 1845, $302=$ spectabilis, $F r$.

TARDCS, B., Hook. Journ., 1845, 56 (Res.), Australia.

Tasmanicus, Berk., Fl. Tasm., 254 (Placo.), Tasmania.

taurinus; Pers., Myc. Eur. ii. $37=$ viscosus, Fr.

tegularis, Lev., Ann. Sci. Nat., 146, $131=$ Trametes.

Tetfairin (Kl.), Fr., Epicr., 451 (Mer.), Mauritius.

TENellus, B. \& Cke, Grev. vi. 81 (Res.), U. States.

TENER, Lev., Ann. Sci. Nat., 1846, 139 (Ino.), Guadeloupe.

TENERRIMUs, B. \& Rav., Grev. i. 65 (Res.), U.' States.

TENoIs, Schw., Am. Bor., 456 (Res.), U. States.

tenuis, B., Ann. Nat. Hist. iii. $382=$ Hexagona.

tenuissimus, Lev., Ann. Sci. Nat., 1844, 188 (Ino.), Java.

TEPHROLEUCDs, Fr., Hym. Eur., 545 (Ano.), U. States, Europe.

TEPHRomelas, Mont., Syll., 155 (Pleur.), Guiana.

TEPhronotes, B., Flor. Tasm., 252 (Ano.), Tasmania, Australia.

TEPBroporus, Mont., Syll., 161 (Res.), Surinam.

terebraxis, B. \& C., Linn. Journ. x. 306 (Pleur.), Cuba.

TERRESTRIs (D.C.), Fr., Hym. Eur., 576 (Res.), U. States, Europe.

terrestris, Sow., t. 387, f. $5=$ bombycinus, Fr.

TEssulatus, Fr., Hym. Eur., 523 (Mes.), Europe.

TESTACEC8, Fr., Hym. Eur., 545 (Ano.), U. States, Europe.

testacers, Lev., Ann. Sci. Nat., 1846, 126 (Pleur.), Brazil.

ThelephoroIdes (Hook.), Fr., Epic., 473 (Ino.), Tropical America.

Tromsoni, B., Hook. Jon?m., 1852, 142 (Placo.), India.

ThwaItesit, B., Hook. Joürn., 1854, 228 (Ino.), Ceylon.

Tilis (Schulz.), Fr., Hym. Eur., 528 (Mes.), Europe.

Todari, Inz., Sic., t. $2=$ snlphureus, Fr.

rovextosus, Fr., Hym. Eur., 530 (Ḿes.), U. States, Eurnpe. 
Toмmstoscs, Rostk. iv. t. 8 (nec Fries) (Mes.), Europe. twratus, Pers., Freyc. Voy. = australis, Fr.

torrilus, Fr., Nova Symb., $73=$ Trametes.

тost's, B., Hook. Journ., 1844, 189 (Placo.), Philippines.

iR.uBLe's (Rostk.), Fr., Hym. Eur., 547 (Ano.), Siberia.

rachodes, Lev., Ann. Sci. Nat., 1844, 192 (Placo.), Java.

rkichy's's, Mont., Syll., 154 (Mes.), Columbia.

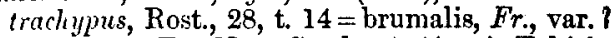

тmichocoma, Fr., Nora Symb., 37 (Ano.), Tahiti.

thicholoya, Mont., Syll., 153 (Mes.), Cuba, Australia, Guiana, Mexico.

trichomallus, M. \& B., Mont., Syll., 163=Trametes.

тиich rous, $B . \&$. C. (Mer.), U. States.

tricolor, Lev., Ann. Sci. Nat., 1846, 134 (Ino.), Brazil.

тuigoxts, Lev., Ann. Sci. Nat., 1846, 125 (Ano.), Java.

triqueter, Fr., Hym. Eur., 565 (Ino.), Europe.

thisticulus, Mont., Ann. Sci. Nat., 1854, 129 (Pleur.), Cayenne.

tristis, Lev., Ann. Sci. Nat., 1846, 126=Trametes.

tristis, Pers., Myc. Eur. ii. 94 (Ino.), Europe.

tropices, Jungh., Fl. Java, 63 (Placo.), Java.

TLBA, B. \& C., Linn. Journ. x. 305 (Mes.), Cuba.

t'meraster, Fr., Hym. Eur., 523 (Mes.), Europe.

tuliercu! osus, Jungh. (fide Lev.) = trachodes, Fr.

TCBrrculosus, Fr., Sys. Myc. i. 380, U. States.

turritus, Pers., Myc. Eur. ii. 117, uncertain.

cDes, Jungh., Fl. Java, 88, Java.

clmarius, Fr., Hym. Eur., 562 (Placo.), Arctic America, U. States, Europe.

umbellatus, Fr., Hym. Eur., 507 (Mer.), U. States, Europe. umbellatus, Viv., t. $28=$ Barrelieri, Fr.

umbilicatus, B., Hook. Journ., 1851, 79 (Mes.), India. umbilicatus, Jungh., Fl. Java = arcularius, $F r$.

cMnoxates, Fr., Nova Symb., 71 (Ino.), Mexico.

imbacoldM, Fr., Epic., 435 (Mes.), Tropics, Mauritius, Cape.

rMirixus, Fr., Hym. Eur., 571 (Res.), U. States, Europe.

umbrinus, Pers., Myc. Eur. ii. $\mathbf{9 3}=$ obliquns, Fr.

undatus, Pers., Myc. Eur. ii. t. 16, f. $3=$ cryptarum, Fr.

cxpigerus, $B$. \& C., Linn. Journ. x. 317 (Ino.), Cuba.

rxdulates (Schw.), Fr., Epic., 460 (Ano.), U. States.

Exguicularis, Fr., Nova Symb., (Pleur.), Mexico.

rxeviformis, Lev., Ann. Sci. Nat., 1846, 129 (Ino.), Java. ungulatus, Bull, t. 491 = fomentarius, Fr.

vxicolon (Schw.), Fr., Epic., 458 (Ano.), U. States.

virrus (P.), Fr., Hym. Eur., 570 (Res.), U. States, Europe.

e rsivus, Fr., Sys. Myyc. i. 361 (Placo.), Brazil, Natal.

Vaimiaxtir, Fr., Hym. Eur., 579 (Res.), Ceylon, Guiana, U. States, Europe.

Valenzuelianus, Mont., Cuba, t. xv. f. 4 (Ino.), Cuba, U. States.

vallatus, Fr., Hook. Journ., 1852, 138 (Pleur.), India.

vaporarius, Fr., Hym. Eur., 579 (Res.), Australia, Cuba, Juan Fernandez,

U. States, Chili, Ceylon, Tasmania, N. Zealand, Europe.

V.ariabilis, B., Hook. Journ., 1856, 193 (Pleur.), Brazil. vitriegatus, Schff., t. $263=$ versicolor, $F r$.

variegatus (Secr.), Fr., Hym. Eur., 563 (Placo.), Europe.

rarioloses, B. \& Br., Linn. Journ. xiv. 52 (Res.), Ceylon.

varics, Fr., Hym. Eur., 535 (Pleur.), Australia, Java, U. States, Siberia, Europe.

vbikTe, Fr., Hym. Eur., 5is (Placn.), India, Europe. 
velierecs, B., Hook. Journ. i. 451 (Ino.), New Ireland. velutinus, Sow., t. $345=$ hispidus, Fr.

velutines, Fr., Hyin. Eur., 568 (Ino.), St Domingo, Java, Cuba, U. States, New Zealand, Brazil, Japan, Philippines, Europe.

velutinus, Fl. Dan., t. $1138=$ resinosus, Fr.

vexclosus, Jungh., $F l$. Jara, 57 (Ino.), Mauritius, Java.

vexustos, $B$., Hook. Journ., 1845, 55 (Ino.), Ceylou, Australia.

verecundus, $B$. \& $C$., Linn. Journ. x. 300 (Ano.), Cube

versalis, Fr., Hym. Eur., 527 (Mes.), Europe.

vericipes, B., Linn. Journ. xvi. 50 (Pleur.), Japan.

VERNICIFLUUS, $B$., Fl. Tasm., 254 (Ino.), Tasmania.

vernicosus, $B$., Hook. Journ., 1856, 175 (Mfes.), Brazil.

verrocolosos (Meyer.), Fr., Ep., 464 (Placo.), Surinam, Columbia. versatilis, B., Fr., Nova Srm., $76=$ Trametes.

versicolor, Fr., Hym. Eur., 568 (Ino.), Cuba, Brazil, U. States, India, Guadeloupe, Java, Tasmania, N. Zealand, Siberia, Cape, Juan Fernandez, Mexico, Australia, Europe.

versicutis, $B$. \& C., Linn. Journ. x. 308 (Ano.), Cuba.

versiforMIs, B., Hook. Journ., 1852, 137 (Pleur.), India. versipellis, Pers., Myc. Eur. ii. 96, uncertain.

versiporus, Pers., Myc. Eur. ii. $105=$ vaporarius, $F$.

vesiculosus, B.\& C., Grev. i. 65 (Res.), U. States.

resparius, B., Ann. Nat. Hist., jii. $323=$ Hexagona Gunnii, $B$.

vespilloneus, B., Hook. Journ., 1856, 199 (Ino.), Brazil.

vibecives, Fr., Fung. Natal, 6 (Pleur.), Natal.

vibratilis, $B$. \& $C$., Liwn. Journ. x. 314 (Ino.), Cuba.

villosus, Fr., Epic., 474 (Ino.), Jamaica.

vinctus, $B$., Ann. Nat. Hist., 1852 (Res.), St Domingo, Cuba, Ceylon.

vivosus, $B$., Ann. Nat. Hist., 1852 (Placo.), Cuba, St Domingo.

violaceus, Fr., Hyrn. Eur., 592 (Res.), U. States, Europe.

VIRELlus, Fr., Hym. Eur., 525 (Mes.), Europe.

virgatcs, $B$. \& C., Linn. Journ. x. 304 (Mes.), Cuba.

virgineus (Schw.), Fr., Epic., 481 (Ino.), U. States.

viridaxs (B. \& Br.), Fr., Hym. Eur., 576 (Res.), Europe.

viscosus, Pers., Myc. Eur. ii. 41 (Mes.), Europe.

vitelinves (Schw.), Fr., Epic., 483 (Res.), U. States.

viticola (Schvo.), Fr., Epic., 482 (Res.), U. States.

vitreus, Fr., Hym. Eur., $57 T$ (Res.), U. States, Europe.

vitratus, $B$., Hook. Journ., 1847, 505 (Ino.), Ceylon.

vivax, B., Hook. Journ., 1862, 140 (Ano.), India.

voluta, Pers., Myc. Eur. ii. 119, uncertain.

volvatus, Peck., 27th Report, 98 (Placo.), U. States.

volganis, Fr., Hym. Eur., 578 (Res.), Mauritius, Cuba, U. States, Ceylon, Java, Brit. North America, Juan Fernandez, Australia, Europe.

volneratus, Lev., Ann. Sci. Nat., 1844, 188 (Ino.), Java.

volPINUs, Fr., Hym. Eur., 565 (Ino.), Europe.

Weddelit, Mont., Ann. Sci. Nat. v. 366 (Mes.), Brazil.

Werimani, Fr., Hym. Eur., 552 (Ano.), Europe.

Wirtaent, Fr., Hym. Eur., 570 (Res.), Europe.

Wightii, Klot., Linn. vii. $200=$ Trametes sinensis, $F r$.

WrNNeI (B. \& Br.), Fr., Hym. Eur., 569 (Ino.), Europe.

Xalapensis, B., Grev. i. 52 (Ino.), Xalapa, U. States(=prolificans, Fr. ?) Xalapensis, Curt., Sill. Journ., $1850=$ elongatus, Fr.

xantholoya, Schwo., Am. Bor., 435 (Res.), U. States, Cuba.

xasthopus, Fr., Epic., 437 (Mes.), India, Surinam, Guiana, Peru, Guinea, Java, Pegu, Philippines, Sumatra, Cochin China, Penang. 
x.мтнсs (P.), Fr., Hym. Eur., 574 (Res.), U. States, Europe. xerajipelixus, Kalch., Grev. iv. 72 (Ino.), Australia. Xeraxticus, B., Hook. Journ., 1852, 161 (Ino.), India. x kroph illaceus, B., Hook. Journ., 1856, 199 (Ino.), India, Brazil. xeropurluds, Berk., Fl. N. Zeal. (Pleur.), N. Zealand. yoilopes (Rostk.), Fr., Hym. Eur., 525 (Mes.), Europe. xulodes, B., Hook. Journ., 1856, 171 (Mes.), Brazil. xrlostrojiatoides, B., Hook. Journ., 1843, 638 (Res.), Brazil. xrlostromatis, Fckl., Sym. Myc., supp. (Res.), Europe.

xylostromeus, Pers., Myc. Eur. ii. $112=$ vitreus, Fr.

Zeylasicus, B., Ann. Nat. Hist., 1843, 377, Ceylon.

Zollingerianus, Lev., Ann. Sci. Nat., 1846, $131=$ Trametes.

zovalis, B., Ann. Nat. Hist. x. 375 (Placo.), India, Bornen, Cuba, Ceylon, Brazil, Mexico, Aru, Philippines, U. States.

zoxatus, Fr., Hym. Eur., 568 (Ino.), Mexico, U. States, Europe.

\section{TRAMETES, Fr.}

Actixopila, Mont., Ann. Sci. Nat. v. 370, Brazil. aCdTA, Lev., Ann. Sci. Nat., 1814, 196, Sumatra. accleifera, B. \& C., Linn. Journ. x. 319, Cuba. ACUPUNCTATA, B., Linn. Journ. xiii. 164, Australia. Ethaloides, Mont., Ann. Sci. Nat. v. 370, Brazil. asibigua, Fr., Nova Symb., 80, Antilles, Gualoupe. ANEIRINa (Fr.), C. \& Q., Clavis. Hym. (Res.), Ceylon, U. States, Europe. atypa, Fr., Nova Symb., $80=$ Polyporus atypus, Lev. A CREA, Berk., Ann. Nat. Hist., 1843, 373.

BaDIA, B., Hook. Journ., 1842, 151, Philippines, Rawak. bala NiNa, Fr., Fung. Natal, 15, Natal.

benzoina, Fr., Epicr., $489=$ Polyporus benzoinus, Fr. Beyrichir, Fr., Linn. Jour. v. 518, Brazil, Philippines. Bicolor, B., Linn. Journ. xvi. 43, Aru.

Boybrcina (Fr.), C. \& Q., Clavis. Hym. (Res.), U. States, Europe.

Bulliardi, Fr., Hym. Eur., 584, Europe.

Campestris (Q.), Fr., Hym. Eur., 585 (Res.), Europe. Captiosa, Mont., Ann. Sci. Nat., 1847, 170, S. Africa. Cextralis, Fr., Nova Symb., 79, Mexico, W. Indies. cingulaata, B., Hook. Journ., 1852, 164, India, Pegu. cinvabarixa, Fr., Hym. Eur., 583, Australia, Ceylon, India, Sumatra, U. States, Arctic America, St Domingo, Costa Rica, Tasmania, Siberia, New Zealand, Pegu, Europe.

circinats, Fr., Fung. Natal, 8, Natal. cogsata, B., Linn. Journ. xvi. 43, Arn. colliculosA, B., Hook. Journ., 1847, Australia, Ceylon, India.

CoNtcla (B.), Fr., Nova Symb., 81, Guiana. coxchats, B., Linn. Journ. xvi. 47 , Philippines. confragosa, Rabh. = Dædalea confragosa, Fr. Crassa, Lev., Ann. Sci. Nat., 1844, 197, Madagascar. crexulata, B., Hook. Journ., 1852, 164, India. Curreri, C. (umbrina, Currey), Pegu.

dermatodes, Leq., Ann. Sci. Nat., 1844, 196, Plilippines.

detonse, Fr. = Polyporus detonsus, Fr.

DeVExa, B., Linn. Journ. xiii. 165. Australia. 
enegaxs, Fr., Epicr., 492, Guadeloupe, W. Indies, Mexico.

нріцові, $F r$., Hym. Eur., 585 (Res.), Europe.

epiterhra, $B$., Linn. Journ. xiii. 165, Australia.

expallexs, Fr., Nora Symb., 79, Guinea.

Feathermaxis, Rav. (sub Polyporus), U. States.

Fibros $\Lambda$, Fr., Epicr., 490, Cuba, N. America, Mexico, A ustralia, Brazil, Demerara, Surinam.

FuscA, Fr., Epicr., 490, Brazil.

FCsCELLA, Lev., in Zoll. Verz., 17, Indian Archipelago.

Gallica, Fr., Hym. Eur., 582, Europe.

gibbosa, Fr., Hym. Eur., 583, Europe, Ceylon, Siberia.

glabrescens, Fr., Nova Symb., $80=$ Polyporus glabrescens, $B$.

Guroniana, Mont., Ann. Sci. Nat. v. 334, Algeria.

HELVOLA, Fr., Epic., 490, Guinea.

hexigovoldes, Fr., Hym. Eur., 585 (Res.), Europe.

HISPIDA (Bagl.), Fr., Hym. Eur., 583, Europe.

hispidela, B. \& C., Linn. Journ. x. 319, Ceylon, Cuba.

HOOKERI, B., Hook. Journ., 1852, 164, India.

hYDNuIDEs, Fr., Epic., 490, Cuba, Brazil, Mauritius, Guiana, Jamaica, St Domingo, Central America, St Thomas, U. States, St Vincents, Demerara, Mexico.

IMMOTATA, B., Hook. Jour., 1852, 165, India.

INCans, Lev., Aun. Sci. Nat., 1844, 196, Philippines, Australia.

incana, Berk. = lactea, $B$.

incoxpITA, Fr., Epic., 490, Guinea, Natal.

INODORA, Fr., Hym. Eur.. 584, Europe.

ISABELlina, Fr., Hym. Eur., 585 (Res.), Europe.

Isidiomes, Fr., Fungi. Natal, 10, India, Natal.

LACTEa (B.), Fr., Nora Symb., 80, U. States.

laCtinea, B., Ann. Nat. Hist. x. 373, Cuba, Ceylon, U. States, Brazil, A ustralia, Admiralty Island.

Leticolor, B., Ann. Nat. Hist., 1843, 374, Ceylon.

LEvis, B., Hook. Jour., 1847, 507, Ceylon.

LANATA, Fr., Epicr., 499, India, Guinea, Natal, Java, Nicobar.

LIGNICOLA, Lasch., in Klotsch. Herb., 1809.

Limitata, $B$. $\&$. $C$., Grev. i. 66 , U. States.

LINDHEIMERI, B.\& $C$. , Grev. i. 66, U. States.

Lobata, B., Hook. Journ., 1851, 84, Ceylon, India.

Ma Rchionica, Mont., Syll., 168, Brazil, Marqueses.

MIexicaNa, B. \& C., Linn. Journ. ix. 423, Mexico.

Micheneri, $\dot{B} . \& \dot{C}$., in $H$ erb. = benzoina, $F r$.

rollis, Fr., Hym. Eur., 585 (Res.), Pluilippines, Australia, Kamtschatka, Mexico, Europe.

Moritziavus, Lev., in Zoll. Verz., Java.

Mosei.eI, B., Linn. Journ. xvi. 40, Tongatabu.

Molleri, B., Linn. Journ. x. 320, Cuba, Australia, Brazil

Natalensis, Fr., Fungi Nat., 13, Natal.

ocellata, B. \& C., Linn. Journ. x. 319, Cuba

occidentalis, Fr., Epic. i. 491, Cuba, Ceylon, India, Mauritiug, Brazil, Guiana, Philippines, Centr. America, U. States, Australia, St Vincent, Admiralty Island, Aru, Guadelonpe, Madagascar, Java, St Dómingo, Antilles. 
udora, Fr., Hym. Eur., 584, Siberia, Europe.

(1)undta, Fr., Hym. Eur., 502, U. States, Siberia, Europe.

olltxis, Buk, Grev. i. 66, U. States.

ozosiondes, B., Hook. Jour., 1852, 166, India.

P.ALACEA, Fr., Epicr., 471, Guinea.

P'innotetri, Lev., Ann. Sci. Nat., 1844, 195, Java.

pirfesi, Fr., Fung. Nalal, 10, Natal, Cape.

Petersil, B. \& C., Grev. i. 66, U. States.

pHEllixs, B., Linn. Jour. xiii. 164, Australia.

I'IN, Fr., Hym. Eur., 582, Australia, U. States, Europe.

Pini, Thum., Myc. Univ. = Poly. pinicola, Fr.

rnotecs, Fr, Fungi Natal, 12, Natal, Cape.

Irotracta, Fr., Hym. Eur., 583, Europe.

IYrrhocreas, B., Linn. Journ. xiji. 164, Australia

jixices, Fr., Nova Symb., 82, Penang.

piks, B. \& C., Linn. Journ. x. 320, Cuba

radiciperda, Hart. Krank. = Poly. annosus, $F r$.

nigida, B. \& M., Ann. Sci. Nat., 1849, 240, Cuba, Brazil, St Domingo,

U. States, Australia.

rcbescess, Fr., Hym. Eur., 584, U. States, Europe.

RcGosi, B. \& Br., Linn. Journ. xiv. 55, Ceylon.

Sagreana, Mont., Syll., 168, Cuba, Guiana.

scalaris, Fr., Fungi Natal, 12, Natal.

sceletos, Fr., Fungi Natal, 14, Natal.

sclerodepsis, B., Hook. Journ., 1856, 236, Brazil.

scleroderma, Fr., Fungi Natal, 15, Natal.

scrobiculata, B., Grev. vi. 70, Australia.

skic M, B., Hook. Journ. vi. 322, U. States.

seriaIIS, Fr., Hym. Eur., 585, U. States, Europe.

serpexs, Fr., Hym. Eur., 586, Siberia, Europe.

sinensis, Fr., Epic., $489=$ Hexagona sinensis.

sixcosa (Fr.), C. \&Q., Clavis. Hym., U. States, Europe.

SpreceI, B., Hook. Journ., 1856, 336, Brazil, Cuba.

Stephensil (B. \& Br.), C. \& Q., Clavis. Hym. (Res.), Europe, U. States, Ceylon.

scaveoless, Fr., Hym. Eur., 584, U. States, Europe.

stberosa, Q., Europe.

tegclarig, Lev., in Zoll. Verz., Java.

TFiproleUCA, B., Hook. Journ., 1852, 185, India.

'Trnrei, B. \& Br., Ann. Nat. Hist. No. 1571 (Res.), Europe.

Torrid, Fr., Epic., 490, Guinea.

Trogir, Fr., Hym. Eur., 583, Europe.

trichomalia, B. \& M. (sub Polyporus), Brazil.

tristis, Lev., in Zoll. Verz., Java.

rubrixa, Fr., Fungi Natal, 13, Natal.

umbrina, Curr., Linn. Trans., 1876, 124=Curreyi, Cke.

rxgclata, B., Linn. Journ. xiii. 165, Australia, India.

versatilis, B., Hook. Journ. i. 450, Borneo, Philippines, Brazil, Cuba,

Australia, U. States.

versiformis, B. \& Br., Linn. Journ. xiv. 56 (Res.), Ceylon.

vittata, Lev., Ann. Sci. Nat., 1844, 196, Sumatra.

Warbergit, Fr., Fungi Natal, ii. Natal. 